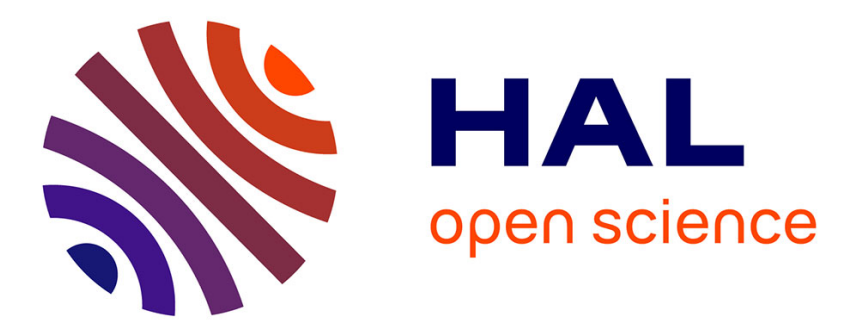

\title{
Identification des paramètres de comportement des sols injectés par analyse inverse d'essais pressiométriques
}

Christophe Dano, Pierre-Yves Hicher, Sylvie Tailliez, Michèle Varjabédian

\section{To cite this version:}

Christophe Dano, Pierre-Yves Hicher, Sylvie Tailliez, Michèle Varjabédian. Identification des paramètres de comportement des sols injectés par analyse inverse d'essais pressiométriques. Revue Française de Génie Civil , 2002, 6 (4), pp.631 - 660. 10.1080/12795119.2002.9692392 hal-01006938

\section{HAL Id: hal-01006938 \\ https://hal.science/hal-01006938}

Submitted on 22 Oct 2016

HAL is a multi-disciplinary open access archive for the deposit and dissemination of scientific research documents, whether they are published or not. The documents may come from teaching and research institutions in France or abroad, or from public or private research centers.
L'archive ouverte pluridisciplinaire HAL, est destinée au dépôt et à la diffusion de documents scientifiques de niveau recherche, publiés ou non, émanant des établissements d'enseignement et de recherche français ou étrangers, des laboratoires publics ou privés.

\section{(c)(1)}

Distributed under a Creative Commons Attribution| 4.0 International License 


\title{
Identification des paramètres de comportement des sols injectés par analyse inverse d'essais pressiométriques
}

\author{
Christophe Dano* — Pierre-Yves Hicher* — Sylvie Tailliez** \\ Michèle Varjabédian***
}

* Laboratoire de Génie Civil de Nantes Saint-Nazaire

Ecole Centrale de Nantes, BP 92101

F-44321 Nantes cedex 3

** Coyne-et-Bellier

9 allée des Barbanniers

F-92632 Gennevilliers

*** Régie Autonome des Transports Parisiens

Département des Infrastructures et Aménagements

40 bis rue Roger Salengro

F-94724 Fontenay-sous-bois

RÉSUMÉ. L'amélioration des propriétés mécaniques d'un sol par injection d'un coulis de nouvelle génération peut être quantifiée soit par des essais de laboratoire, soit par des essais in situ. Il a été déduit des essais de laboratoire qu'un modèle de comportement élastique linéaire avec un critère de plasticité non associé de Mohr-Coulomb permet de donner une approximation du comportement mécanique des sols injectés. On propose d'identifier les paramètres de ce modèle par analyse inverse d'essais pressiométriques. La procédure est basée sur l'expression analytique de la courbe pressiométrique donnée par (Yu et Houlsby, 1991) et un algorithme d'optimisation de Newton-Gauss. Les résultats sont comparés avec les paramètres déduits des essais triaxiaux en laboratoire.

ABSTRACT. The improvement of the mechanical properties of a soil by injection of a new generation grout can be assessed either by laboratory tests or by field tests. It has been deduced from laboratory tests that a linear elastic - perfectly plastic model with a non associated MohrCoulomb failure criterion enables to roughly reproduce the mechanical behaviour of grouted sands. We propose in this paper to identify the parameters of this model by inverse analysis of pressuremeter tests. The procedure is based on the analytical expression of the pressuremeter curve given by (Yu and Houlsby, 1991) and a Newton-Gauss optimization algorithm. The results are then compared with the values of the parameters determined in the laboratory.

MOTS-CLÉS : analyse inverse, essais pressiométriques, sols injectés.

KEYWORDS: Inverse analysis, pressuremeter tests, grouted soils. 


\section{Introduction}

L'injection des sols consiste à introduire, sous pression, un coulis fluide qui, en faisant prise, permet de rendre moins perméable et de renforcer le milieu poreux traversé. Ce procédé, utilisé dans le domaine des travaux souterrains, permettrait de réduire le coût des soutènements si l'amélioration des propriétés mécaniques du sol en place était rationnellement et systématiquement prise en compte. La diversité des facteurs dont dépend la qualité du traitement (nature, porosité, granulométrie, anisotropie du sol; nature, rhéologie, granulométrie du coulis; paramètres d'injection) n'a pas permis, jusqu'à présent, d'établir des corrélations à partir desquelles la rigidité et la résistance du sol injecté pourraient être déterminées et prises en compte dans les calculs de conception des ouvrages. Une action de recherche, initiée par la Régie Autonome des Transports Parisiens (RATP) en 1995, en collaboration avec les sociétés spécialisées dans l'injection, tente de remédier à ce problème.

Un vaste programme expérimental en laboratoire a abouti, dans un premier temps, à décrire les principales caractéristiques du comportement mécanique des sols injectés à court et à définir des facteurs d'amélioration des propriétés mécaniques des sols injectés par rapport aux sols en place (Biarez et al., 1998; Tailliez, 1998 ; Dano, 2001).

Dans un deuxième temps, plusieurs campagnes d'essais pressiométriques in situ ont permis de justifier les travaux de laboratoire. On présente ainsi les facteurs d'améliorations des caractéristiques pressiométriques dans trois cas réels (Biarez et al., 1998 ; Tailliez, 1998 ; Dano, 2001). A terme, les bureaux d'études ne pourront toutefois pas se satisfaire de la seule donnée de ces caractéristiques pressiométriques.

Ainsi, les calculs de conception requièrent souvent l'identification des paramètres de modèle de comportement. On montre donc, dans un troisième temps, la procédure retenue pour identifier les paramètres du modèle élastique plastique parfait par analyse inverse d'essais pressiométriques.

\section{Comportement mécanique des sols injectés}

Les gels de silice, aujourd'hui interdits pour des raisons de protection de l'environnement et de tenue dans le temps, ont été remplacés par des coulis dits de nouvelle génération, de nature minérale (ils sont désignés par $\mathrm{CM}$ dans ce document) ou à base de ciment fine mouture (désignés par $\mathrm{CC}$ ), aux performances similaires en termes d'injectabilité. Ils peuvent imprégner des sols granulaires dont la perméabilité est comprise entre $10^{-3}$ et $10^{-5} \mathrm{~m} / \mathrm{s}$. Les coulis utilisés ici, déclinés sous plusieurs formulations, sont injectés dans des terrains granulaires divers, depuis des sables fins jusqu'aux alluvions anciennes de la Seine, secs ou saturés, dont la granulométrie (figure 1) a été écrêtée à un centimètre. Le comportement mécanique 
de ces sols après injection est analogue à celui observé sur les sols traités par d'autres types de coulis (Tailliez, 1998), en particulier les gels de silice, ou sur les sols obtenus par malaxage, tels que les mortiers (Dupas et Pecker, 1979) ou les sables traités au ciment (Clough et Sitar, 1981). Biarez et al. (1998), Tailliez (1998) et Dano (2001) ont constaté que :

- l'injection de coulis engendre une augmentation appréciable de la résistance et de la rigidité du terrain (figure 2);

- le sol injecté présente un comportement volumique contractant-dilatant avec un domaine de contractance réduit et un taux de déformation volumique accru par rapport à celui du terrain initial (figure 2) ;

- le comportement est plus ou moins fragile en fonction de la nature et de la teneur en liant du coulis, du sol et de la contrainte moyenne (figure 2);

- pour les coulis à base de ciment ou de nature minérale, contrairement aux gels de silice, la vitesse de sollicitation n'a que peu d'influence sur la réponse mécanique du sol injecté (figure 3). Ceci permet de comparer des résultats d'essais triaxiaux et d'essais pressiométriques qui ne sont pas nécessairement réalisés avec une même vitesse de chargement.

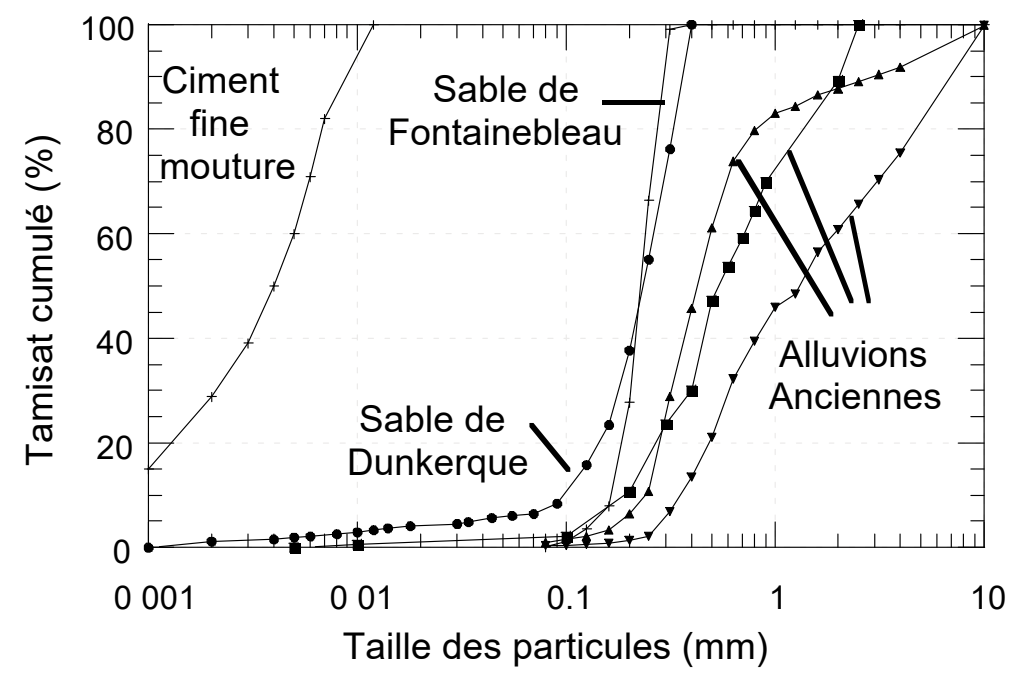

Figure 1. Courbes granulométriques des sols étudiés (Tailliez, 1998 ; Dano, 2001)

La résistance et la rigidité des sols injectés dépendent principalement :

- de la teneur en liant du coulis,

- de la contrainte moyenne,

- de la densité et des caractéristiques du sol granulaire initial.

La teneur en liant du coulis est définie par le rapport massique $\mathrm{C} / \mathrm{E}$ (rapport de la quantité de liant sur la quantité d'eau de gâchage) préalablement choisi en fonction de la nature des travaux à réaliser (étanchéité, renforcement mécanique). 

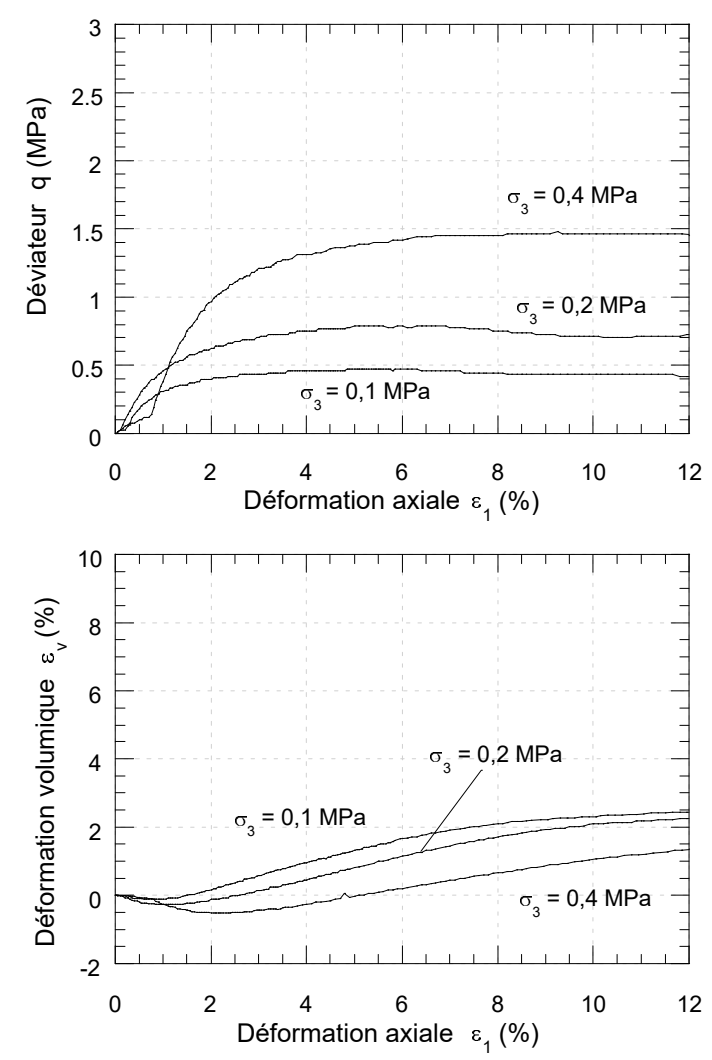

Sable de Seine
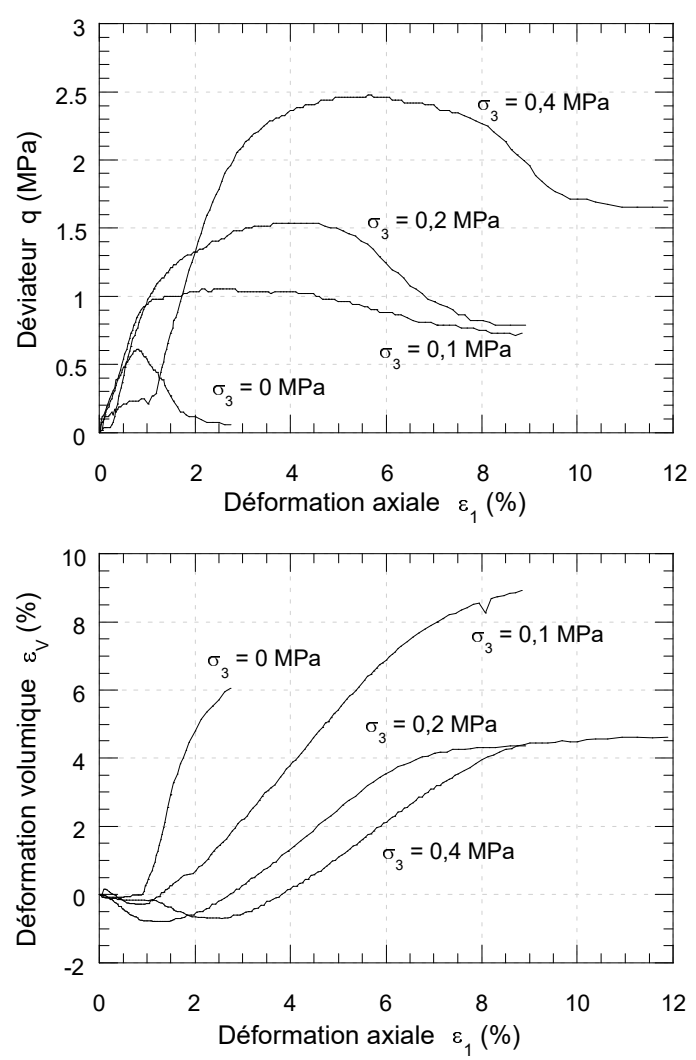

Sable de Seine + Coulis de ciment fine mouture $\mathrm{CC}, \mathrm{C} / \mathrm{E}=0,07$
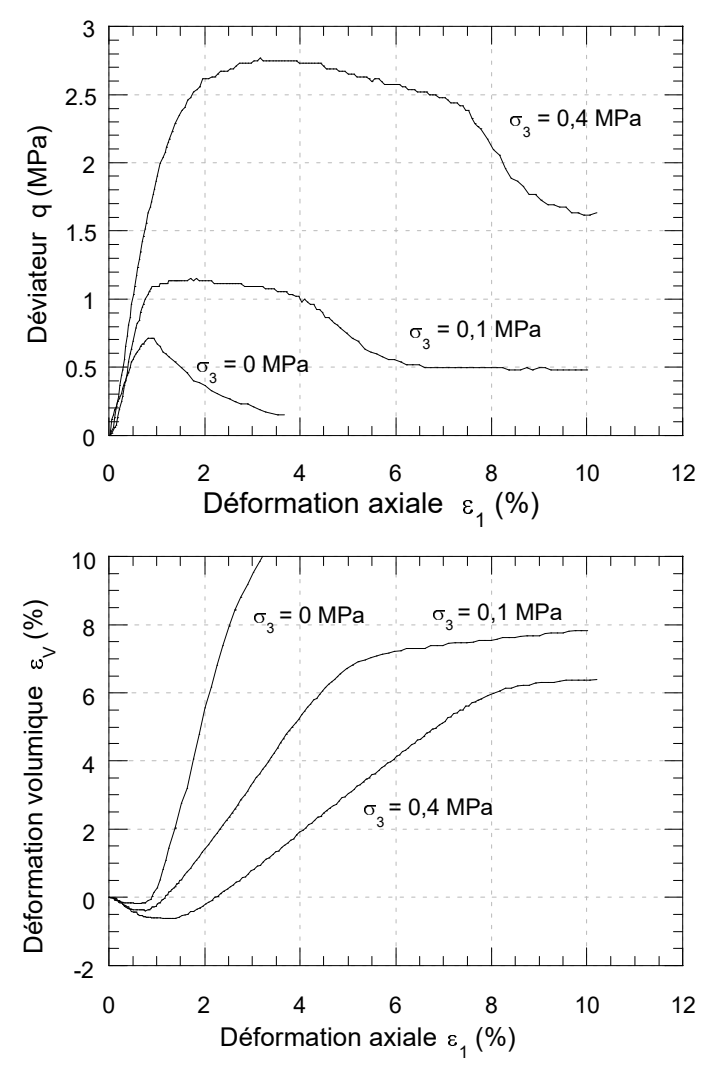

Sable de Seine + Coulis de ciment minéral $\mathrm{CM}, \mathrm{C} / \mathrm{E}=0,056$
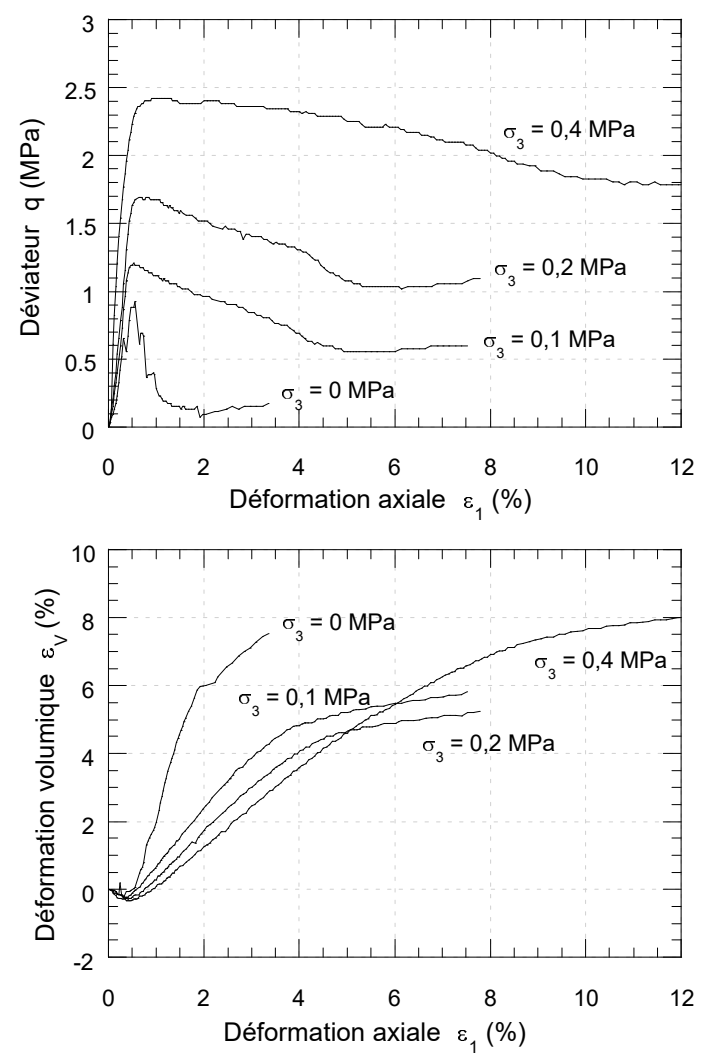

Sable de Fontainebleau + Coulis de ciment fine mouture $\mathrm{CC}, \mathrm{C} / \mathrm{E}=0,07$

Figure 2. Essais triaxiaux sur sables vierges et sables injectés (Tailliez, 1998) 


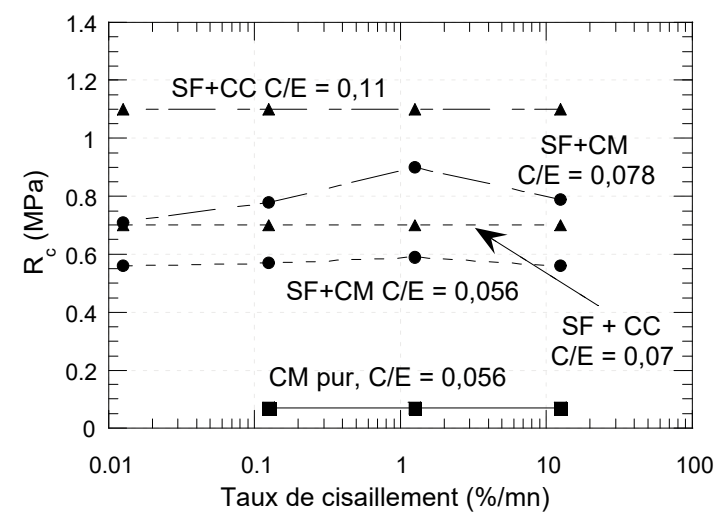

Figure 3. Influence de la vitesse de sollicitation sur la résistance en compression simple des sols injectés (Tailliez, 1998)

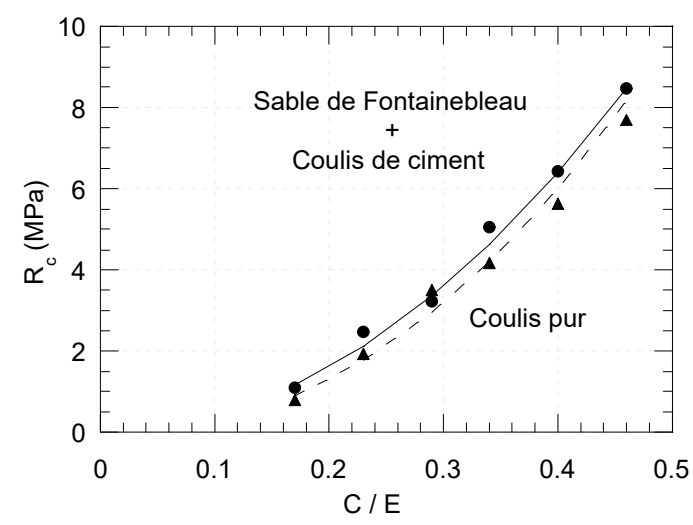

Figure 4. Evolution de la résistance en compression simple avec le rapport $\mathrm{C} / \mathrm{E}$ (Dano, 2001)

Ainsi, sur la base d'essais de compression simple sur des échantillons de sable de Fontainebleau (noté SF) mis en place avec un indice de densité relative $\mathrm{I}_{d}$ de $78 \%$ [1] et injectés en laboratoire par des coulis de ciment de rapports $\mathrm{C} / \mathrm{E}$ croissants, on propose la relation formelle suivante pour la résistance en compression simple $\mathrm{R}_{\mathrm{c}}$ (figure 4) (Dano, 2001) :

$$
\mathrm{R}_{\mathrm{c}}=\left[\lambda_{\mathrm{c}}+\lambda_{\mathrm{s}}\right] \times\left(\frac{\mathrm{C}}{\mathrm{E}}\right)^{\left(\mathrm{N}_{\mathrm{c}}-\mathrm{N}_{\mathrm{s}} \times \mathrm{I}_{\mathrm{d}}\right)} \quad \text { avec } \mathrm{I}_{\mathrm{d}}=\frac{\gamma_{\max }}{\gamma} \times \frac{\gamma-\gamma_{\min }}{\gamma_{\max }-\gamma_{\min }}
$$

où $I_{d}$ est l'indice de densité relative $(\%) ;\left(\lambda_{c}, N_{c}\right)$ les paramètres caractérisant le coulis pur ; $\left(\lambda_{\mathrm{s}}, \mathrm{N}_{\mathrm{s}}\right)$ les paramètres caractérisant le sol ; $\gamma$ le poids volumique du sol ; $\gamma_{\min }$ et $\gamma_{\max }$ respectivement le poids volumique minimal et le poids volumique maximal $\mathrm{du}$ sol. Le paramètre $\lambda_{\mathrm{s}}$ dépend de l'indice de densité relative, du coefficient d'uniformité du sol et prend une valeur nulle pour $I_{d}=0 \%$.

Dans le cas du sable de Fontainebleau $\left(\mathrm{I}_{\mathrm{d}}=78 \%, \mathrm{C}_{\mathrm{u}}=1,4\right)$ injecté avec un coulis à base de ciment fine mouture, on obtient (figure 4), dans l'intervalle des rapports $\mathrm{C} / \mathrm{E}=[0 ; 0,5]:$

$$
\mathrm{R}_{\mathrm{c}}=40 \times\left(\frac{\mathrm{C}}{\mathrm{E}}\right)^{2}
$$

Cependant, la donnée de la résistance en compression simple est souvent insuffisante, même si elle constitue un bon indicateur de l'amélioration apportée par l'injection. Les calculs d'ouvrages requièrent de plus en plus souvent l'identification des paramètres des modèles de comportement introduits dans les codes de calcul numérique. Parmi ces modèles, le modèle élastique linéaire isotrope avec un critère 
de plasticité de Mohr-Coulomb (Mestat, 1997) est encore largement utilisé dans les bureaux d'études. C'est dans cet esprit que l'on a cherché à interpréter les résultats des essais triaxiaux. On reporte, dans le plan de Mohr, les états de contraintes correspondant à la résistance maximale des échantillons. On s'aperçoit alors que les enveloppes intrinsèques pour le sable vierge de tout traitement et le sable injecté sont linéaires dans le domaine des contraintes de compression étudiées et parallèles entre elles (figure 5). Par conséquent, l'angle de frottement interne $\varphi^{\prime}$ n'est pratiquement pas modifié par l'imprégnation d'un coulis. Par contre, la cohésion c' est notablement augmentée. La valeur de la cohésion peut être estimée à partir de la relation [1] puisque :

$$
\mathrm{R}_{\mathrm{c}}=\frac{2 \mathrm{c}^{\prime} \cdot \cos \varphi^{\prime}}{1-\sin \varphi^{\prime}}
$$

d'où :

$$
\mathrm{c}^{\prime}=\frac{1-\sin \varphi^{\prime}}{2 \times \cos \varphi^{\prime}} \times\left[\left(\lambda_{\mathrm{c}}+\lambda_{\mathrm{s}}\right) \times\left(\frac{\mathrm{C}}{\mathrm{E}}\right)^{\left(\mathrm{N}_{\mathrm{c}}-\mathrm{N}_{\mathrm{s}} \times \mathrm{I}_{\mathrm{d}}\right)}\right]
$$

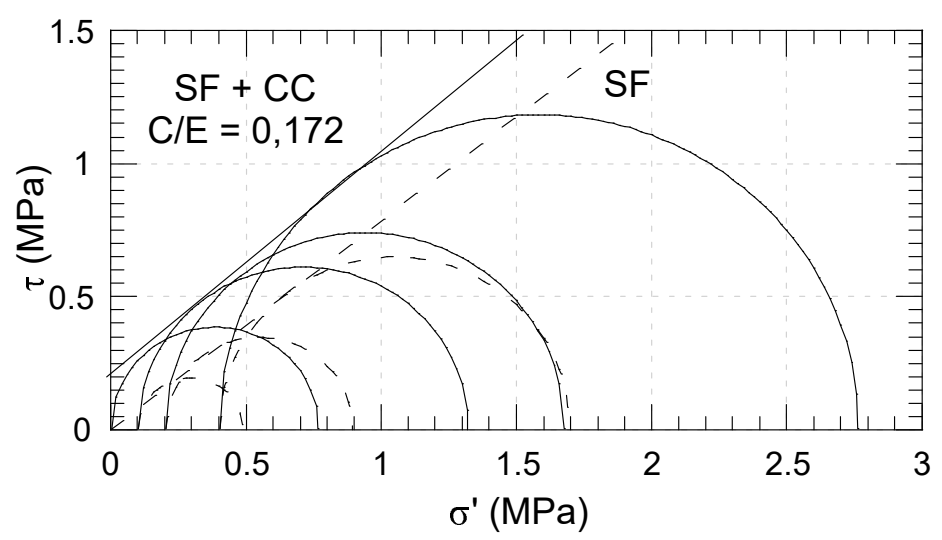

Figure 5. Enveloppes intrinsèques pour le sable vierge et le sable injecté

La loi d'écoulement plastique doit être considérée comme non associée afin de représenter correctement l'évolution des déformations volumiques (Baguelin et al., 1978 ; Kasdi, 1994) pour les sols non traités moyennement denses à fortement denses et pour les sols injectés, pour lesquels le comportement volumique est dilatant. Enfin, comme le montre la figure 2, la courbe contrainte-déformation apparaît, en première approximation, linéaire sur un domaine assez large avant de s'incurver au voisinage du pic de contraintes. L'hypothèse d'élasticité linéaire semble donc être appropriée. 
En conclusion, on adopte par la suite un modèle élastique parfaitement plastique non associé avec une loi élastique linéaire et isotrope et un critère de plasticité de Mohr-Coulomb. Ce modèle est défini par cinq paramètres : le module d'Young E, le coefficient de Poisson v, l'angle de frottement interne $\varphi$ ', la cohésion c' et l'angle de dilatance $\psi$. Ce modèle est un bon compromis pour donner une approximation des propriétés générales du comportement des sols injectés et pour conduire à une expression analytique de la courbe pressiométrique.

\section{Expression de la courbe pressiométrique}

\subsection{Interprétation conventionnelle des essais pressiométriques}

Les conditions in situ ne se prêtent généralement pas à des prélèvements aisés et non remaniés des échantillons pour des essais de laboratoire ultérieurs. Il est donc préférable de réaliser des essais en place. L'essai pressiométrique, décrit dans les normes NF P 94-110, est l'un des plus couramment pratiqués. On en déduit généralement un paramètre de déformabilité du sol, le module pressiométrique Ménard noté $\mathrm{E}_{\mathrm{m}}$, et un paramètre de résistance, la pression limite notée $\mathrm{p}_{\mathrm{l}}$.

On reporte dans le tableau 1 les résultats de trois campagnes d'essais pressiométriques réalisés à des profondeurs comprises entre 3 à 10 mètres :

- la première au port autonome de Dunkerque dans un sable marin, fin homogène, injecté par un coulis minéral pour un traitement d'étanchéité (Biarez et al., 1998 ; Tailliez, 1998);

- la deuxième sur le chantier Madeleine du métro Météor à Paris dans des alluvions anciennes injectées avec un gel de silicate pour un renforcement mécanique du terrain (Biarez et al., 1998 ; Tailliez, 1998);

- la troisième sur le chantier Saint-Lazare du prolongement de la ligne Météor dans des alluvions anciennes injectées avec un coulis de ciment fine mouture pour un renforcement mécanique du terrain (Dano, 2001).

Chaque campagne comprend des essais pressiométriques avant injection et des essais pressiométriques après injection. Les rapports $\mathrm{E}_{\mathrm{m}} / \mathrm{p}_{\mathrm{l}}$, compris entre 6 et 10 , sont typiques des sols granulaires normalement serrés à très serrés (Fascicule 62, Titre V). On peut alors déterminer les facteurs d'amélioration (rapport de la valeur d'une grandeur mécanique considérée après injection sur sa valeur avant injection) sur le module pressiométrique $\mathrm{E}_{\mathrm{m}}{ }^{*}$, sur le module pressiométrique calculé sur un cycle de déchargement-rechargement $\mathrm{E}_{\mathrm{mc}}{ }^{*}$ et sur la pression limite $\mathrm{p}_{1}{ }^{*}$. Ces facteurs, calculés sur les valeurs moyennes des caractéristiques sans distinction de la profondeur, dépendent principalement de l'objet du traitement (renforcement ou étanchement) (tableau 1). Ils sont aussi très proches des facteurs d'amélioration du module élastique et du déviateur maximal, pour une contrainte moyenne donnée, déterminés à partir d'essais triaxiaux en laboratoire. 


\subsection{Principe de l'analyse inverse}

Les paramètres des modèles de comportement, introduits dans les codes de calcul numériques, sont classiquement déterminés au moyen d'essais de laboratoire, mais pour les raisons invoquées précédemment, leur identification à partir d'essais in situ est envisageable. Une première méthode d'identification consiste à utiliser les corrélations empiriques reliant les caractéristiques pressiométriques et les paramètres du modèle de comportement (Combarieu, 1995), mais cette méthode montre rapidement ses limites. Une seconde méthode, dont le principe est exposé dans (Shahrour et al., 1995), consiste à identifier les paramètres d'un modèle par analyse inverse de l'ensemble des points formant la courbe pressiométrique. Il s'agit alors de minimiser l'écart entre la courbe expérimentale et le résultat d'une simulation numérique ou d'un calcul analytique de l'essai pressiométrique, par des itérations successives portant sur les paramètres du modèle de comportement. On présente ici le cas de l'analyse inverse à partir d'une expression analytique de l'essai pressiométrique.

\begin{tabular}{|c|c|c|c|c|c|c|}
\hline Chantier & \multicolumn{2}{|c|}{$\begin{array}{c}\text { Port Autonome } \\
\text { Dunkerque } \\
\end{array}$} & \multicolumn{2}{|c|}{$\begin{array}{c}\text { Métro Madeleine } \\
\text { Paris } \\
\end{array}$} & \multicolumn{2}{|c|}{$\begin{array}{c}\text { Métro } \mathrm{S}^{\mathrm{t}} \text {-Lazare } \\
\text { Paris }\end{array}$} \\
\hline Objet du traitement & \multicolumn{2}{|c|}{ Etanchéité } & \multicolumn{2}{|c|}{ Renforcement } & \multicolumn{2}{|c|}{ Renforcement } \\
\hline Terrain & \multicolumn{2}{|c|}{$\begin{array}{l}\text { Sable marin fin, } \\
\text { homogène et propre }\end{array}$} & \multicolumn{2}{|c|}{$\begin{array}{l}\text { Alluvions Anciennes, } \\
\text { Sables et graviers, } \\
\gamma=15,7 \mathrm{kN} / \mathrm{m}^{3}\end{array}$} & \multicolumn{2}{|c|}{$\begin{array}{l}\text { Alluvions Anciennes, } \\
\text { Sables et graviers, } \\
\gamma=15,7 \mathrm{kN} / \mathrm{m}^{3}\end{array}$} \\
\hline Coulis & \multicolumn{2}{|c|}{ Minéral } & \multicolumn{2}{|c|}{ Gel de silice } & \multicolumn{2}{|c|}{ Ciment fine mouture } \\
\hline \multirow{3}{*}{$\begin{array}{c}\text { Module } \\
\text { pressiométrique } \\
\mathrm{E}_{\mathrm{m}}(\mathrm{MPa}) \\
\mathrm{E}_{\mathrm{m}}^{*} \\
\end{array}$} & $\begin{array}{c}\text { Avant } \\
\text { injection }\end{array}$ & $\begin{array}{c}\text { Après } \\
\text { injection }\end{array}$ & $\begin{array}{c}\text { Avant } \\
\text { injection }\end{array}$ & $\begin{array}{c}\text { Après } \\
\text { injection }\end{array}$ & $\begin{array}{c}\text { Avant } \\
\text { injection }\end{array}$ & $\begin{array}{c}\text { Après } \\
\text { injection }\end{array}$ \\
\hline & 17 à 28 & 39 à 85 & 16 à 98 & 60 à 176 & 1 à 17 & 32 à 69 \\
\hline & \multicolumn{2}{|c|}{2,2} & \multicolumn{2}{|c|}{4} & \multicolumn{2}{|c|}{5,9} \\
\hline \multirow{3}{*}{$\begin{array}{c}\text { Module cyclique } \\
\mathrm{E}_{\mathrm{mc}}(\mathrm{MPa}) \\
\mathrm{E}_{\mathrm{mc}}^{*} \\
\end{array}$} & $\begin{array}{c}\text { Avant } \\
\text { injection }\end{array}$ & $\begin{array}{c}\text { Après } \\
\text { injection }\end{array}$ & $\begin{array}{l}\text { Avant } \\
\text { injection }\end{array}$ & $\begin{array}{c}\text { Après } \\
\text { injection }\end{array}$ & $\begin{array}{c}\text { Avant } \\
\text { injection }\end{array}$ & $\begin{array}{c}\text { Après } \\
\text { injection }\end{array}$ \\
\hline & - & - & 52 à 71 & 565 & 12 à 63 & 63 à 130 \\
\hline & & \multicolumn{2}{|c|}{9,2} & \multicolumn{2}{|c|}{3,2} \\
\hline \multirow{3}{*}{$\begin{array}{c}\text { Pression limite } \\
\mathrm{p}_{1}(\mathrm{MPa}) \\
\mathrm{p}_{1}^{*}\end{array}$} & $\begin{array}{c}\text { Avant } \\
\text { injection }\end{array}$ & $\begin{array}{c}\text { Après } \\
\text { injection }\end{array}$ & $\begin{array}{c}\text { Avant } \\
\text { injection }\end{array}$ & $\begin{array}{c}\text { Après } \\
\text { injection }\end{array}$ & $\begin{array}{c}\text { Avant } \\
\text { injection }\end{array}$ & $\begin{array}{c}\text { Après } \\
\text { injection }\end{array}$ \\
\hline & 2,6 à 4,0 & 4,6 à 7,2 & 1,0 à 3,3 & 3,7 à 8,2 & 0,3 à 2,5 & 3,3 à 6,6 \\
\hline & \multicolumn{2}{|c|}{1,4} & \multicolumn{2}{|c|}{2,5} & \multicolumn{2}{|c|}{4,4} \\
\hline
\end{tabular}

Tableau 1. Facteurs d'amélioration des caractéristiques pressiométriques 


\subsection{Etablissement de l'expression analytique de la courbe pressiométrique}

La courbe pressiométrique (figure 6) relie la déformation à la paroi du forage $\mathrm{U}_{\mathrm{a}} / \mathrm{a}$ (où $\mathrm{U}_{\mathrm{a}}$ est le déplacement de la paroi et «a » le rayon de la sonde pressiométrique) ou, de manière équivalente, les variations de volume de la sonde, à la pression $\mathrm{p}$ dans cette même sonde. D'un point de vue théorique, il s'agit de résoudre le problème mécanique de l'expansion d'une cavité cylindrique dans un milieu semi-infini. On fait également l'hypothèse de déformation plane dans le plan horizontal $\left(\varepsilon_{z z}=0\right.$, où $\mathrm{z}$ est la direction verticale). Elle est justifiée par l'utilisation de cellules de garde de part et d'autre de la sonde pressiométrique. On suppose que la sonde se dilate dans un milieu semi-infini, tridimensionnel, isotrope et homogène. Le comportement du sol est représenté par le modèle élastique linéaire-plastique parfait non associé avec un critère de plasticité de Mohr-Coulomb.

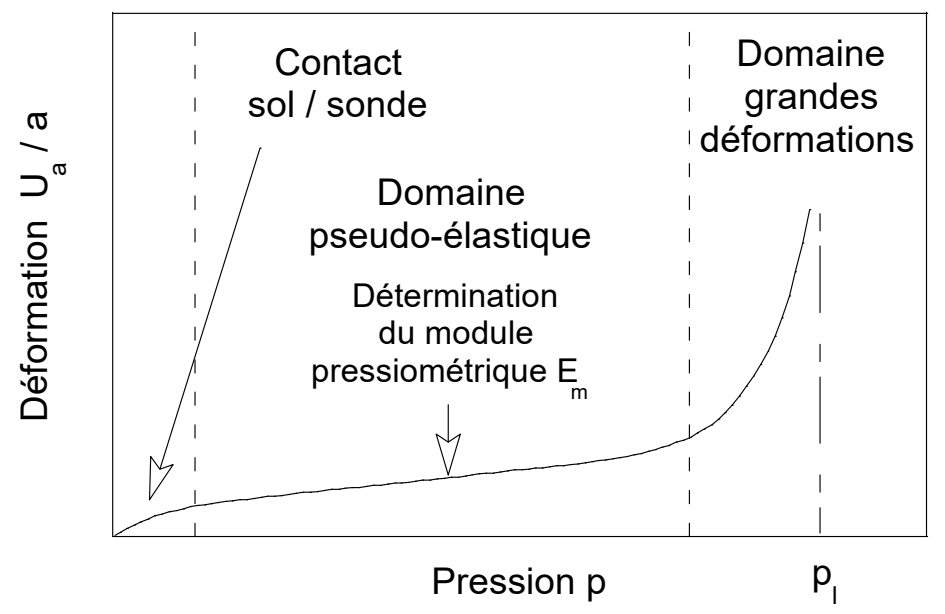

Figure 6. Exemple de courbe pressiométrique

Dans le système de coordonnées cylindriques $(\mathrm{r}, \mathrm{z})$, on combine :

- les équations d'équilibre dans le plan horizontal [5a] et dans le plan vertical [5b] :

$$
\begin{aligned}
& \frac{\mathrm{d}_{\mathrm{r}}}{\mathrm{dr}} \frac{\mathrm{r} \theta}{\mathrm{r}} 0 \\
& \frac{\mathrm{d}_{\mathrm{z}}}{\mathrm{dz}}
\end{aligned}
$$

où est le poids volumique du sol; ${ }_{r},{ }_{\theta}$ et ${ }_{z}$ respectivement les contraintes radiales, orthoradiales et verticales ; 
- les conditions aux limites à la paroi du forage [6a] et à l'infini où on considère que le sol n'est perturbé ni par le forage, ni par le chargement :

$$
\begin{aligned}
& \sigma_{r}(a)=-p \\
& \lim _{r \rightarrow \infty} \sigma_{r}=-p_{0}
\end{aligned}
$$

où $\mathrm{p}_{0}$ est la pression des terres au repos ;

- les relations de compatibilité pour une analyse en petites déformations :

$$
\begin{aligned}
& \varepsilon_{\mathrm{r}}(\mathrm{r})=\frac{\mathrm{dU}(\mathrm{r})}{\mathrm{dr}} \\
& \varepsilon_{\theta}(\mathrm{r})=\frac{\mathrm{U}(\mathrm{r})}{\mathrm{r}}
\end{aligned}
$$

- les relations de compatibilité pour une analyse en grandes déformations (Yu et Houlsby, 1991) :

$$
\begin{aligned}
& \varepsilon_{\mathrm{r}}(\mathrm{r})=\operatorname{Ln}\left(\frac{\mathrm{dr}}{\mathrm{dr}_{0}}\right) \\
& \varepsilon_{\theta}(\mathrm{r})=\operatorname{Ln}\left(\frac{\mathrm{r}}{\mathrm{r}_{0}}\right)
\end{aligned}
$$

où $r$ est la valeur actualisée du rayon ;

- le modèle de comportement élastique linéaire et isotrope (loi de Hooke définie par l'équation [9]), plastique parfaite avec un critère de plasticité [10] non associé. Les déformations plastiques se déduisent de la fonction potentiel plastique [11] :

$$
\begin{aligned}
& \underset{\underline{\sigma}}{=}=\lambda \times \operatorname{Tr}(\underline{\underline{\varepsilon}}) \underline{\underline{\mathrm{I}}}+2 \mu \times \underline{\underline{\varepsilon}} \\
& \mathrm{f}\left(\sigma_{\mathrm{ij}}\right)=\left|\sigma_{3}-\sigma_{1}\right|-\left(\sigma_{3}+\sigma_{1}\right) \cdot \sin \varphi^{\prime}-2 \mathrm{c}^{\prime} \cdot \cos \varphi^{\prime} \\
& \mathrm{g}\left(\sigma_{\mathrm{ij}}\right)=\left|\sigma_{3}-\sigma_{1}\right|-\left(\sigma_{3}+\sigma_{1}\right) \cdot \sin \psi+\mathrm{g}_{0}
\end{aligned}
$$

où $\lambda$ et $\mu$ sont les coefficients de Lamé et $\mathrm{g}_{0}$ une constante. 
Les incréments de pression initiaux sont d'amplitude faible et entraînent une déformation élastique du sol. L'équation de la courbe pressiométrique, dans le domaine élastique, s'écrit :

$$
\frac{\mathrm{U}_{\mathrm{a}}}{\mathrm{a}}=\frac{\mathrm{p}-\mathrm{p}_{0}}{2 \mathrm{G}}
$$

où $\mathrm{G}$ est le module de cisaillement du sol.

Dès que la pression dépasse la pression $\mathrm{p}_{\mathrm{pl}}$ donnée par l'équation [13], un anneau plastique se développe concentriquement au forage :

$$
\mathrm{p}_{\mathrm{pl}}=\mathrm{c}^{\prime} \cdot \cos \varphi^{\prime}+\mathrm{p}_{0} \times\left(1+\sin \varphi^{\prime}\right)
$$

L'analyse classique en petites déformations (Monnet et Khlif, 1994 ; Monnet et Chemaa, 1995) conduit, dans le cas d'un sol frottant et cohérent, à la courbe pressiométrique suivante dans le domaine plastique :

$$
\frac{\mathrm{U}_{\mathrm{a}}}{\mathrm{a}}=\frac{\mathrm{c}^{\prime} \cdot \cos \varphi^{\prime}+\mathrm{p}_{0} \times \sin \varphi^{\prime}}{2 \mathrm{G}} \times\left[(1+\sin \psi) \times\left(\frac{\mathrm{c}^{\prime} \cdot \cos \varphi^{\prime}+\left(\mathrm{p}_{0}+\Delta \mathrm{p}\right) \times \sin \varphi^{\prime}}{\left(\mathrm{c}^{\prime} \cdot \cos \varphi^{\prime}+\mathrm{p}_{0} \times \sin \varphi^{\prime}\right) \times\left(1+\sin \varphi^{\prime}\right)}\right)^{\frac{1+\sin \varphi^{\prime}}{\sin \varphi^{\prime} \times(1+\sin \psi)}}-\sin \psi\right]
$$

en supposant que les déformations élastiques sont constantes dans le domaine plastique et égales à la déformation élastique atteinte au début du palier de plasticité parfaite. On suppose également qu'une seule zone plastique se développe : elle est provoquée par le déviateur $\mathrm{q}=\left|\sigma_{\mathrm{r}}-\sigma_{\theta}\right|$ entre les contraintes radiale $\sigma_{\mathrm{r}}$ et orthoradiale $\sigma_{\theta}$, la contrainte verticale restant la contrainte intermédiaire. Si tel n'était pas le cas, une seconde zone plastique pourrait se développer, provoquée par le déviateur $\mathrm{q}=\left|\sigma_{\mathrm{r}}-\sigma_{\theta}\right|$ (Monnet et Khlif, 1994 ; Monnet et Chemaa, 1995).

Les hypothèses de petites déformations et de déformations élastiques négligeables dans le domaine plastique ne sont pas nécessairement justifiées. On a donc comparé l'analyse en petites déformations [14] avec l'analyse en grandes déformations proposée par (Yu et Houlsby, 1991), qui donnent l'équation suivante de la courbe pressiométrique dans le domaine plastique :

$$
\frac{\mathrm{U}_{\mathrm{a}}}{\mathrm{a}}=\left\{\frac{\mathrm{R}^{-\chi}}{(1-\delta)^{1+\beta / \beta}-\left(\frac{\chi}{\eta}\right) \times \Lambda_{1}(\mathrm{R}, \xi)}\right\}^{\frac{\beta}{1+\beta}}-1
$$


avec :

$$
\begin{gathered}
R=\frac{(1+\alpha) \times[Y+p \times(\alpha-1)]}{\alpha \cdot(1+\alpha) \times\left[Y+p_{0} \times(\alpha-1)\right]} \quad Y=\frac{2 c^{\prime} \cdot \cos \varphi^{\prime}}{1-\sin \varphi^{\prime}} \\
\alpha=\frac{1+\sin \varphi^{\prime}}{1-\sin \varphi^{\prime}} \quad \beta=\frac{1+\sin \psi}{1-\sin \psi} \\
\delta=\frac{Y+(\alpha-1) \times p_{0}}{2 \beta \times(1+\alpha)} \quad \chi=\frac{\alpha \times(1+\beta)}{\beta \times(\alpha-1)} \\
\eta=\exp \left\{\frac{(1+\beta) \times(1-2 v) \times(1-v)}{\beta \times(\alpha-1)} \times\left[\alpha \beta+1-\frac{v \times(\alpha+\beta)}{1-v}\right]\right. \\
\Lambda_{1}(x, y)=\sum_{n=0}^{\infty} \frac{y^{n}}{n !(n-\omega)}\left[x^{n-\omega}-1\right] \text { pour } n \neq \omega .
\end{gathered}
$$

La figure 7 représente les solutions en petites et en grandes déformations pour le jeu de données de référence indiqué dans le tableau 2. Il apparaît que 1'écart est assez important et que, pour pouvoir identifier en particulier les paramètres de rupture, il convient de choisir la solution en grandes déformations. La solution proposée par (Yu et Houlsby, 1991) a donc été programmée dans un tableur qui possède un outil d'optimisation basée sur l'algorithme de Newton-Gauss. Ce type d'algorithme a déjà été utilisé, avec succès, dans des procédures d'analyse inverse d'essais de laboratoire (Shao et al., 1991).

\subsection{Sensibilité de la courbe pressiométrique aux paramètres du modèle de comportement}

L'algorithme de Newton-Gauss affecte la même pondération à tous les paramètres optimisés. Cependant, plusieurs études montrent que tous les paramètres n'ont pas la même influence sur la courbe pressiométrique (Kasdi, 1994). Une étude de sensibilité permet de mettre en avant les paramètres-clés et d'orienter le calcul d'optimisation.

Une courbe de référence est calculée à partir de l'équation [15] et à partir du jeu de paramètres de référence donné dans le tableau 2. La valeur de chacun des paramètres, représentative des sols couramment rencontrés en géotechnique, est successivement changée de $30 \%$, toute chose égale par ailleurs. La figure 8 représente les courbes pressiométriques obtenues. 


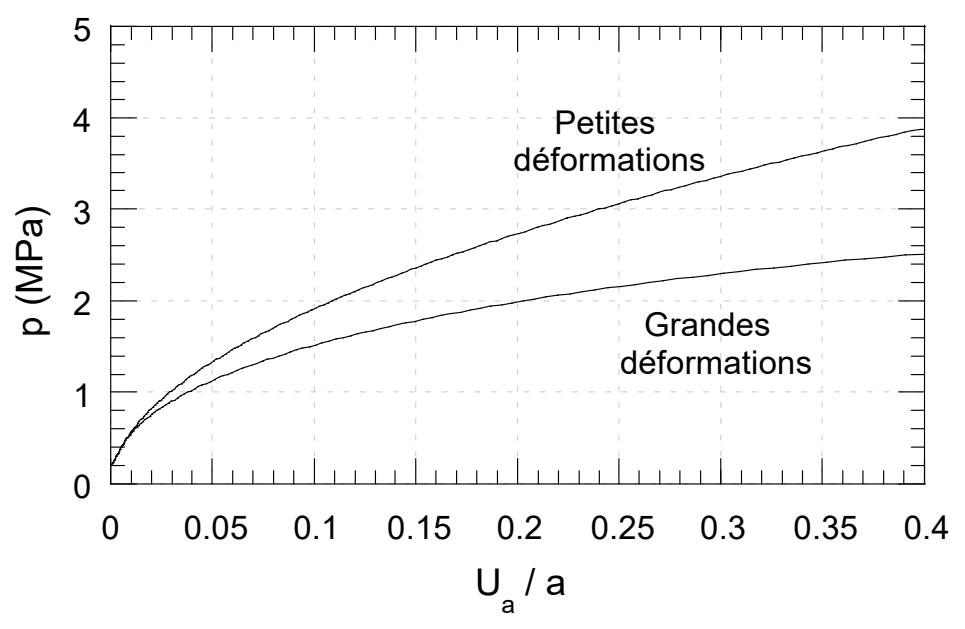

Figure 7. Comparaison de la courbe pressiométrique en petites et grandes déformations

\begin{tabular}{|cccccc||}
\hline $\begin{array}{c}\mathrm{E} \\
\mathrm{MPa}\end{array}$ & $v$ & $\begin{array}{c}\varphi^{\prime} \\
\text { degrés }\end{array}$ & $\begin{array}{c}\psi \\
\text { degrés }\end{array}$ & $\begin{array}{c}\mathrm{c}^{\prime} \\
\mathrm{kPa}\end{array}$ & $\begin{array}{c}\mathrm{p}_{0} \\
\mathrm{kPa}\end{array}$ \\
\hline 50 & 0,25 & 40 & 15 & 100 & 200 \\
\hline
\end{tabular}

Tableau 2. Jeu de paramètres de référence

Tous les paramètres, excepté le coefficient de Poisson dont l'effet est négligeable, ont, à des degrés différents, la même influence qualitative sur l'évolution de la courbe pressiométrique : une augmentation de la valeur de ces paramètres se traduit par une rigidification apparente du matériau. Par ailleurs, si le module élastique affecte l'ensemble de la courbe pressiométrique, l'angle de frottement interne et, à un degré moindre la cohésion et l'angle de dilatance, contrôle la forme de la courbe dans le domaine des grandes déformations. On note aussi que la valeur de la pression des terres au repos doit être considérée comme un paramètre du modèle tant elle influe sur l'allure de la courbe. Pour conclure, les paramètres principaux sont le module élastique, l'angle de frottement interne et la pression des terres au repos, les paramètres secondaires la cohésion et l'angle de dilatance.

\subsection{Compléments sur la technique d'optimisation}

L'optimisation simultanée de tous les paramètres, du fait de leur effet qualitatif semblable sur la courbe pressiométrique et du couplage de certains d'entre eux (l'angle de frottement interne et l'angle de dilatance par exemple), peut conduire à une divergence des calculs ou tout simplement à des valeurs erronées. Il est donc 
nécessaire de réduire le nombre de variables à optimiser simultanément, soit en fixant la valeur de certains des paramètres par l'expérimentation en laboratoire, soit en les corrélant entre eux, soit encore en définissant des contraintes sur les paramètres. En dépit de ces précautions, certains calculs d'optimisation convergent vers des valeurs physiquement non acceptables à cause de l'existence de minima locaux : le calcul s'arrête alors avant que le minimum absolu ne soit atteint. Une solution préconisée par (Zentar, 1999) consiste à procéder à deux calculs d'optimisation avec des jeux de paramètres initiaux très différents et de s'assurer qu'ils convergent vers la même solution.

\section{Procédure d'identification des paramètres du modèle de comportement}

La procédure décrite ci-dessous, qui précise celles proposées par (Schnaid et al., 1998) pour le cas des sols frottants et cohérents et (Haberfield et Johnston, 1990) pour les roches de faible résistance mécanique, vise à réduire le nombre de variables à optimiser par l'utilisation de corrélations empiriques qui permettent de lier les valeurs de certains paramètres, de résultats bibliographiques et par la réalisation d'essais pressiométriques en deux étapes :

- une première série d'essais pressiométriques comprenant au moins un cycle de déchargement-rechargement est réalisée avant injection dans le sol pulvérulent, à différentes profondeurs. Six paramètres définissent le matériau non injecté : $p_{0 v}, E_{v}$, $v_{v}, \varphi^{\prime}, \mathrm{c}_{v}, \psi_{v}$;

- une seconde série d'essais pressiométriques comprenant au moins un cycle de déchargement-rechargement est réalisée après injection, à différentes profondeurs. Six nouveaux paramètres définissent la matériau injecté : $\mathrm{p}_{0 \mathrm{i}}, \mathrm{E}_{\mathrm{i}}, v_{\mathrm{i}}, \varphi_{\mathrm{i}}{ }_{\mathrm{i}}, \mathrm{c}^{\prime}{ }_{\mathrm{i}}, \psi_{\mathrm{i}}$.

Les douze paramètres sont alors déterminés de la manière exposée ci-dessous.

\section{Pressions des terres au repos $p_{{ }_{0 v}}$ et $p_{{ }_{0 i}}$}

La valeur de la pression des terres au repos, notée $\mathrm{p}^{{ }_{0 \mathrm{v}}}$ pour le sol vierge et $\mathrm{p}^{\prime}{ }_{0 \mathrm{i}}$ pour le sol injecté, déterminée au début du palier pseudo-élastique de la courbe pressiométrique, est souvent entachée d'erreurs expérimentales dues à la phase de déchargement et au remaniement induit par la réalisation du forage. Souvent, ces valeurs sont nettement exagérées par rapport à l'appréciation que l'on peut en avoir sur le site. Aussi, si la phase de reconnaissance géotechnique ne fournit pas de données fiables quant aux valeurs de la pression des terres au repos, on les calcule à partir du poids des terrains surmontant la couche de sol étudié :

$$
\mathrm{p}_{0}^{\prime}=\mathrm{K}_{0} \times \sigma^{\prime}{ }_{\mathrm{v}}=\mathrm{K}_{0} \times \sum_{\mathrm{i}} \gamma_{\mathrm{i}}^{\prime} \times \mathrm{z}_{\mathrm{i}}
$$

où $\sigma^{\prime}$, est la contrainte verticale effective $; \gamma_{i}^{\prime}$ le poids déjaugé de la couche de sol $\mathrm{i} ; \mathrm{z}_{\mathrm{i}}$ son épaisseur et $\mathrm{K}_{0}$ le coefficient de pression des terres au repos. 


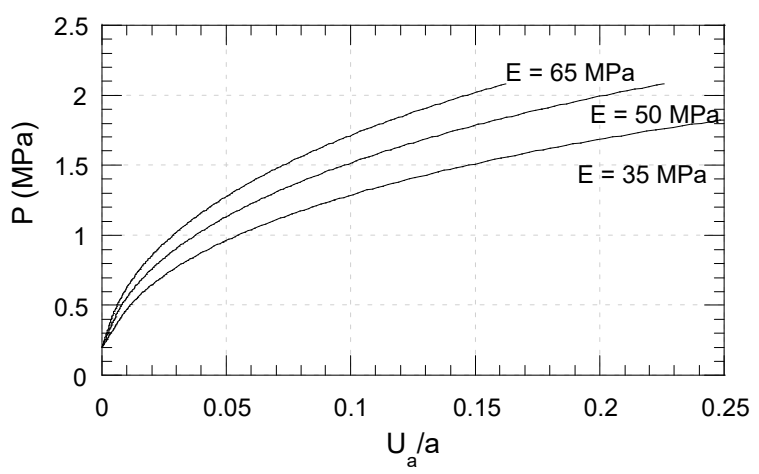

Influence de E.

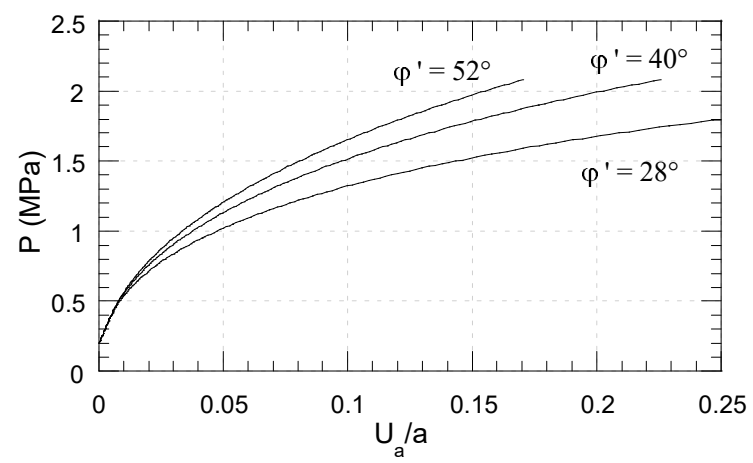

Influence de $\varphi^{\prime}$.

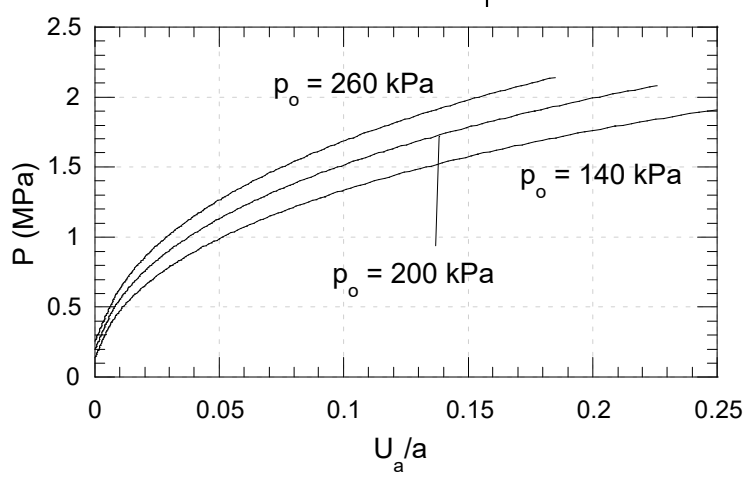

Influence de $\mathrm{p}_{0}$.

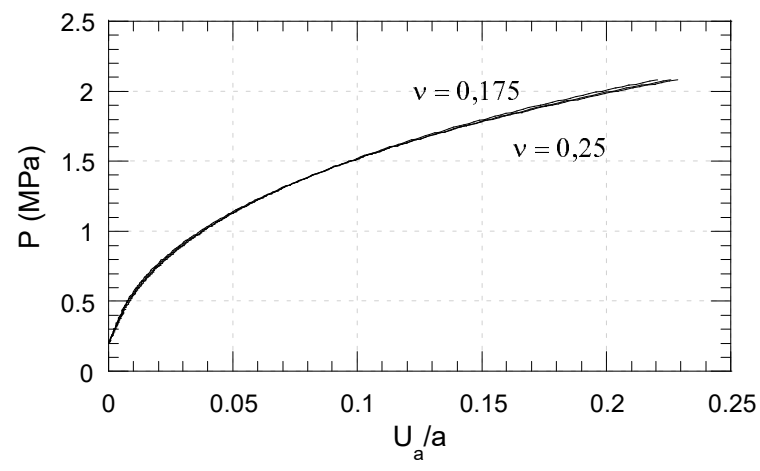

Influence de $v$.

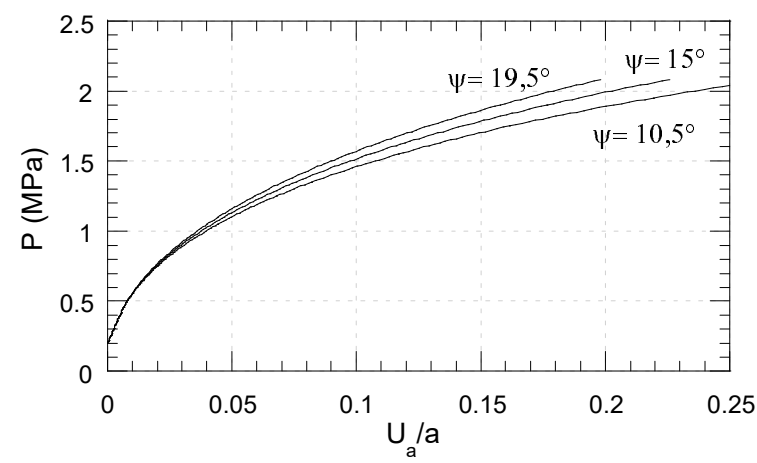

Influence de $\psi$.

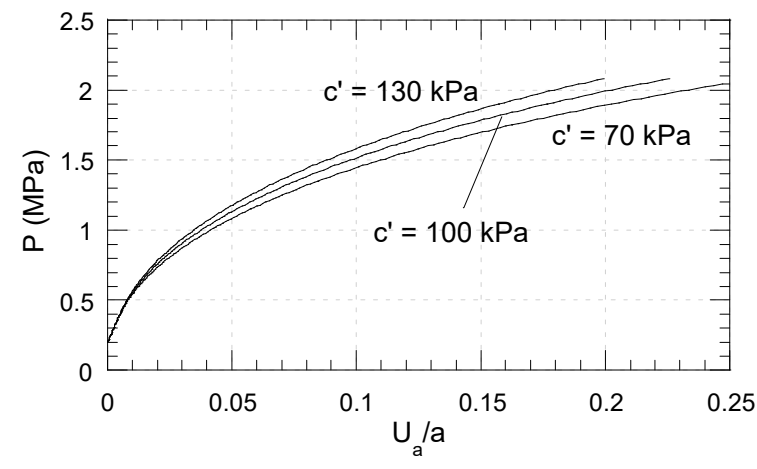

Influence de c'.

Figure 8. Etude de sensibilité

La relation [15] suppose un état de contraintes initial isotrope dans un matériau isotrope. Aussi, sauf indication contraire, on considère, dans la suite de ce document, que le coefficient de pression des terres au repos est égal à $1\left(\mathrm{~K}_{0}=1\right)$. Cette hypothèse n'est toutefois que rarement vérifiée. En effet, la contrainte verticale est souvent supérieure à la contrainte horizontale initiale. Par conséquent, les équations [14] et [15] ne sont pas valables tant que la contrainte verticale n'est pas la contrainte intermédiaire. De même, le remaniement lors du sondage perturbe le début de la courbe pressiométrique. C'est pourquoi on ne tiendra pas compte des premiers points expérimentaux de la courbe pressiométrique, comme proposé par (Cambou et Bahar, 1993). La procédure d'optimisation n'est appliquée que sur la partie de la courbe pressiométrique correspondant à une déformation à la paroi $\mathrm{U}_{\mathrm{a}} / \mathrm{a}$ supérieure à $1,5 \%$. 
Etant donné que, d'une manière générale, l'influence du coefficient de Poisson est négligeable, les paramètres $v_{v}$ (avant injection) et $v_{\mathrm{i}}$ (après injection) ne peuvent pas être significativement optimisés puisqu'une large plage de valeurs peut convenir. Les valeurs couramment observées en géotechnique varient entre 0 et 0,5 . Les essais de laboratoire ont montré que les valeurs du coefficient de Poisson pour les sols non traités ou pour les sols injectés sont généralement comprises entre 0,2 et 0,3. Dans la suite, les valeurs du coefficient de Poisson pour le sol vierge et le sol injecté sont choisies égales à 0,25 .

Modules élastiques $E_{v}$ et $E_{i}$

Il est recommandé d'intégrer dans la procédure de réalisation des essais pressiométriques au moins un cycle de déchargement-rechargement. Ce cycle montre souvent une réponse linéaire du sol sur laquelle la valeur des modules de cisaillement $\mathrm{G}_{\mathrm{v}, \text { cycle }}$ (avant injection) et $\mathrm{G}_{\mathrm{i}, \text { cycle }}$ (après injection) est déterminée :

$$
\mathrm{G}_{\text {cycle }}=\left.\frac{\Delta \mathrm{p}}{\Delta\left(\frac{\mathrm{U}_{\mathrm{a}}}{\mathrm{a}}\right)}\right|_{\text {cycle }}
$$

Cependant, les cycles de décharge-recharge sont généralement exécutés lorsque la déformation dans le sol a déjà atteint $10^{-4}$ ou $10^{-3}$. Aussi, il convient de corriger les valeurs $\mathrm{G}_{\text {cycle }}$ de l'effet du niveau de déformations ou du niveau de contraintes pour obtenir les valeurs du module de cisaillement $G_{v}$ ou $G_{i}$ du sol au début de l'essai pressiométrique. On retient l'expression proposée par (Bellotti et al., 1989) dans le cas des matériaux purement frottants :

$$
\begin{aligned}
& G_{\mathrm{v}}=G_{\mathrm{v}, \text { cycle }}\left(\frac{\sigma_{\mathrm{h} 0}^{\prime}}{\sigma_{\mathrm{av}}^{\prime}}\right)^{\mathrm{n}} \\
& \sigma_{\mathrm{av}}^{\prime}=\sigma_{\mathrm{h} 0}^{\prime}+\alpha_{\mathrm{b}} \times\left(\mathrm{p}_{\mathrm{c}}^{\prime}-\sigma_{\mathrm{h} 0}^{\prime}\right)
\end{aligned}
$$

où $\sigma^{\prime}{ }_{\mathrm{h} 0}$ est la contrainte horizontale moyenne à l'état initial ; $\mathrm{p}_{\mathrm{c}}$ la pression dans la sonde pressiométrique au moment du déchargement ; $\alpha_{b}$ un facteur de réduction qui dépend de la pression des terres au repos $\mathrm{p}_{0}$, de la pression $\mathrm{p}_{\mathrm{c}}$ et de l'angle de frottement interne $\varphi^{\prime} ; \mathrm{n}$ un coefficient compris entre 0,4 et 0,6. (Bellotti et al., 1989) précisent que la valeur du facteur $\alpha_{b}$ est proche de 0,2 . 
Dans le cas des sols injectés, les travaux de (Tailliez, 1998) et de (Dano, 2001) montrent une faible dépendance du module tangent à l'origine des courbes effortdéformation vis-à-vis de la contrainte moyenne. Sur la base de cette constatation, le module de cisaillement après injection $\mathrm{G}_{\mathrm{i}}$, sauf mention contraire, ne subit pas de correction $\left(\mathrm{G}_{\mathrm{i}}=\mathrm{G}_{\mathrm{i}, \text { cycle }}\right)$. Le module élastique dérive alors du module de cisaillement correspondant par la relation générale :

$$
\mathrm{E}=2 \mathrm{G} \times(1+v)
$$

Le module élastique avant injection $\mathrm{E}_{\mathrm{v}}$ est ainsi identifié en tenant compte de la correction contenue dans l'équation [18], en considérant une valeur du facteur $\alpha_{b}$ égale à 0,2 et une valeur de l'exposant $\mathrm{n}$ égale à 0,5 .

\section{Angles de dilatance $\psi_{v}$ et $\psi_{i}$}

Pour les sables vierges de tout traitement, l'angle de dilatance $\psi_{\mathrm{v}}$ est souvent corrélé à l'angle de frottement interne $\varphi^{\prime}{ }_{v}$ (Hughes et al., 1977 ; Bolton, 1986 ; Vaid et Sasitharan, 1992).

On retient la corrélation suivante, représentative de nombreux sables (Dano, 2001) :

$$
\psi_{\mathrm{v}}=\varphi_{\mathrm{v}}^{\prime}-30^{\circ}
$$

D'autres corrélations, plus complexes, ont été proposées dans la littérature (Bolton, 1986 ; Kasdi, 1994) pour tenir compte de l'effet de la densité (tableau 3).

De telles corrélations ne semblent pas exister pour les sols injectés. Le paramètre $\psi_{\mathrm{i}}$ est optimisé avec la contrainte, déduite des essais triaxiaux en laboratoire, selon laquelle l'angle de dilatance du sol injecté est compris entre 0 et 30 degrés mais au moins égal à l'angle de dilatance du sol vierge $\left(\psi_{\mathrm{i}} \geq \psi_{\mathrm{v}}\right)$.

Cependant, il faut noter que l'angle de dilatance prend une définition différente dans le cas de l'essai triaxial axisymétrique et dans le cas de l'essai pressiométrique en déformation plane (Carter et al., 1986). La comparaison directe des angles de dilatance sur des chemins de chargement triaxiaux ou en déformation plane est donc à proscrire.

\section{Cohésions $c^{\prime}{ }_{v}$ et c'}

Pour les sols pulvérulents, la cohésion $\mathrm{c}^{\prime}{ }_{\mathrm{v}}$ est généralement proche de zéro. Aussi, la valeur c ${ }^{\prime}{ }_{v}$ peut être fixée à une valeur comprise entre 0 et $20 \mathrm{kPa}$, laissée à l'appréciation de l'expérimentateur en fonction de l'observation de la nature du matériau. 


\begin{tabular}{||c|cc|c||}
\hline Densité & $\begin{array}{c}\varphi^{\prime} \\
\text { degrés }\end{array}$ & $\begin{array}{c}\psi \\
\text { Degrés }\end{array}$ & $\chi=\psi / \varphi^{\prime}$ \\
\hline Lâche & $30-33$ & $0-8$ & 0,15 \\
Moyennement dense & $33-39$ & $8-12$ & 0,25 \\
Dense & $39-46$ & $12-16$ & 0,30 \\
\hline
\end{tabular}

Tableau 3. Corrélation entre l'angle de frottement interne et l'angle de dilatance (Kasdi, 1994)

Pour les sols injectés, la cohésion c' ${ }_{i}$ est optimisée en même temps que l'angle de dilatance $\psi_{\mathrm{i}}$. En effet, des calculs préalables ont montré l'absence apparente de couplage. La valeur de la cohésion, qui dépend de l'objet du traitement, des propriétés du coulis et du sol, peut être estimée à partir de la relation [1], moyennant quelques essais de compression simple en laboratoire. Les valeurs de cohésion couramment constatées sont comprises entre 200 et $500 \mathrm{kPa}$ pour un traitement de consolidation et proches de $100 \mathrm{kPa}$ pour un traitement d'étanchéité.

Lorsque le calcul d'optimisation de la cohésion est achevé, il faut vérifier que la valeur de la taille du domaine élastique (ou, de manière équivalente, la valeur de la pression seuil $\mathrm{p}_{\mathrm{p}}$, dépendant de la cohésion) n'a pas été modifiée, auquel cas le calcul d'optimisation doit être repris.

Angles de frottement interne $\varphi^{\prime}{ }_{v}$ et $\varphi^{\prime}{ }_{i}$

Dans le cas des sols pulvérulents, l'angle de frottement interne $\varphi^{\prime}$ veste la seule variable à optimiser si l'angle de dilatance $\psi_{\mathrm{v}}$ est corrélé et si le module élastique $\mathrm{E}_{\mathrm{v}}$ est déterminé sur un cycle de déchargement-rechargement. On ajoute une contrainte supplémentaire : l'angle de frottement interne $\varphi^{\prime}{ }_{v}$ doit être compris entre 25 et 50 degrés.

Les essais de laboratoire ont montré que l'imprégnation d'un sol poreux par un coulis n'entraînait pas ou peu de modification de la valeur de l'angle de frottement interne, ce qui peut être expliqué par le fait que la pénétration du coulis dans les interstices se fait sans modification de la structure interne du sol (Cambefort, 1967). Aussi, contrairement aux cas des injections de densification du sol ou des injections se produisant avec des claquages importants, l'angle de frottement interne du sol injecté $\varphi^{\prime}{ }_{i}$ est pris égal à l'angle de frottement interne du sol avant injection $\left(\varphi^{\prime}{ }_{\mathrm{v}}=\varphi_{\mathrm{i}}{ }^{\prime}\right)$.

\section{Applications}

\subsection{Essais pressiométriques en laboratoire sur du sable vierge}

Dans un premier temps, on a cherché à évaluer la fiabilité de la méthode en l'appliquant à des essais pressiométriques réalisés, en chambre de calibration, dans 
du sable d'Hostun purement frottant (Mokrani, 1991). Le sol est mis en place par pluviation autour de la sonde pressiométrique. Une pression appliquée autour de l'échantillon (1,20 $\mathrm{m}$ de diamètre et 1,50 $\mathrm{m}$ de hauteur) permet de représenter l'effet de la profondeur.

Trois essais indicés 8,14 et 16 dont les caractéristiques sont reportées dans le tableau 4 sont soumis à la procédure d'optimisation. L'hypothèse d'une contrainte verticale $\sigma_{v}^{\prime}$ intermédiaire n'est pas justifiée, ce qui induit une mauvaise simulation du début des courbes pressiométriques. En accord avec la procédure, la cohésion est nulle et le coefficient de Poisson est fixé à une valeur de 0,25. Le module élastique noté $\mathrm{E}_{\mathrm{p}}$ (tableau 4) est mesuré sur un cycle de déchargement-rechargement, pendant l'essai pressiométrique, à un faible niveau de déformation. On retient également le module tangent $\mathrm{E}_{\text {triax }}$ déterminé sur des essais triaxiaux conduits dans les mêmes conditions de contrainte latérale et de densité que les essais pressiométriques. On corrige toutefois le module $\mathrm{E}_{\text {triax }}$ de l'effet du chemin de contraintes. Le module corrigé, noté $\mathrm{E}_{\mathrm{dp}}$, est alors :

$$
E_{d p} \frac{E_{\text {triax }}}{1-v^{2}}
$$

Finalement, l'optimisation ne concerne plus que l'angle de frottement interne puisque l'angle de dilatance est corrélé (tableau 5). Quelle que soit la corrélation établie entre ces deux paramètres, les calculs convergent vers une valeur de l'angle de frottement interne proche de celle obtenue en laboratoire pour les essais triaxiaux, sauf pour l'essai 8 (tableau 5, figure 9). Plusieurs raisons peuvent être avancées pour expliquer la différence observée pour cet essai réalisé dans un sable dense : la faible valeur de la pression des terres au repos $\mathrm{p}_{0}$, l'incapacité du modèle à décrire l'éventuel radoucissement post-pic, l'écart pouvant exister entre la valeur de l'angle de frottement interne sur un chemin de chargement axisymétrique et sur un chemin de chargement en déformation plane (écart d'autant plus important que la densité du matériau est importante) (Lee, 1970), le choix de la valeur du module élastique.

Si on maîtrise correctement les valeurs de la pression des terres au repos et du module élastique, les calculs conduisent à des valeurs physiquement plausibles de l'angle de frottement interne, du même ordre de grandeur que les valeurs obtenues en laboratoire.

\begin{tabular}{||c|cccccccc||}
\hline \hline Essai & $\begin{array}{c}\sigma^{\prime} \\
\mathrm{kPa}\end{array}$ & $\begin{array}{c}\mathrm{p}_{0} \\
\mathrm{kPa}\end{array}$ & $\begin{array}{c}\gamma \\
\mathrm{kN} / \mathrm{m}^{3}\end{array}$ & $\begin{array}{c}\mathrm{I}_{\mathrm{d}} \\
\%\end{array}$ & $\begin{array}{c}\mathrm{E}_{\mathrm{p}} \\
\mathrm{MPa}\end{array}$ & $\begin{array}{c}\mathrm{E}_{\text {triax }} \\
\mathrm{MPa}\end{array}$ & $\chi=\psi / \varphi^{\prime}$ & $\begin{array}{c}\varphi^{\prime} \\
\text { degrés }\end{array}$ \\
\hline 8 & 400 & 135 & 16,2 & 84 & 256 & 76 & 0,3 & 41,9 \\
14 & 200 & 84 & 14,4 & 41 & 25 & 26 & 0,15 & 35,4 \\
16 & 500 & 130 & 14,9 & 54 & 79 & 57 & 0,25 & 34 \\
\hline
\end{tabular}

Tableau 4. Caractéristiques des essais en chambre de calibration 


\begin{tabular}{|c|c|c|c|c|}
\hline Essai & Calcul & Contrainte ajoutée & Corrélation $\varphi^{\prime} / \psi$ & $\begin{array}{c}\text { Variable } \\
\text { optimisée }\end{array}$ \\
\hline \multirow{3}{*}{8} & $(8-1)$ & $\mathrm{p}_{0}=135 \mathrm{kPa}, \mathrm{E}=\mathrm{E}_{\mathrm{p}}=256 \mathrm{MPa}$ & $\psi=\varphi^{\prime}-30^{\circ}$ & $\varphi^{\prime}{ }_{\text {opt }}=37,9^{\circ}$ \\
\hline & $(8-2)$ & $\mathrm{p}_{0}=135 \mathrm{kPa}, \mathrm{E}=\mathrm{E}_{\mathrm{dp}}=81,1 \mathrm{MPa}$ & $\psi=\varphi^{\prime}-30^{\circ}$ & $\varphi^{\prime}{ }_{\text {opt }}=52,5^{\circ}$ \\
\hline & $(8-3)$ & $\mathrm{p}_{0}=135 \mathrm{kPa}, \mathrm{E}=\mathrm{E}_{\mathrm{dp}}=81,1 \mathrm{MPa}$ & $\psi=\varphi^{\prime} \times 0,3$ & $\varphi_{\mathrm{opt}}^{\prime}=58,4^{\circ}$ \\
\hline \multirow{3}{*}{14} & $(14-1)$ & $\mathrm{p}_{0}=84 \mathrm{kPa}, \mathrm{E}=\mathrm{E}_{\mathrm{p}}=25 \mathrm{MPa}$ & $\psi=\varphi^{\prime}-30^{\circ}$ & $\varphi^{\prime}{ }_{\mathrm{opt}}=33,9^{\circ}$ \\
\hline & $(14-2)$ & $\mathrm{p}_{0}=84 \mathrm{kPa}, \mathrm{E}=\mathrm{E}_{\mathrm{p}}=25 \mathrm{MPa}$ & $\psi=\varphi^{\prime} \times 0,15$ & $\varphi^{\prime}{ }_{\mathrm{opt}}=33,2^{\circ}$ \\
\hline & $(14-3)$ & $\mathrm{p}_{0}=84 \mathrm{kPa}, \mathrm{E}=\mathrm{E}_{\mathrm{dp}}=27,7 \mathrm{MPa}$ & $\psi=\varphi^{\prime}-30^{\circ}$ & $\varphi^{\prime}{ }_{\mathrm{opt}}=33,1^{\circ}$ \\
\hline \multirow{3}{*}{16} & $(16-1)$ & $\mathrm{p}_{0}=130 \mathrm{kPa}, \mathrm{E}=\mathrm{E}_{\mathrm{p}}=79 \mathrm{MPa}$ & $\psi=\varphi^{\prime}-30^{\circ}$ & $\varphi^{\prime}{ }_{\mathrm{opt}}=33,4^{\circ}$ \\
\hline & $(16-2)$ & $\mathrm{p}_{0}=130 \mathrm{kPa}, \mathrm{E}=\mathrm{E}_{\mathrm{dp}}=60,8 \mathrm{MPa}$ & $\psi=\varphi^{\prime}-30^{\circ}$ & $\varphi^{\prime}{ }_{\mathrm{opt}}=35,5^{\circ}$ \\
\hline & $(16-3)$ & $\mathrm{p}_{0}=130 \mathrm{kPa}, \mathrm{E}=\mathrm{E}_{\mathrm{dp}}=60,8 \mathrm{MPa}$ & $\psi=\varphi^{\prime} \times 0,25$ & $\varphi^{\prime}{ }_{\mathrm{opt}}=33,6^{\circ}$ \\
\hline
\end{tabular}

Tableau 5. Calculs d'optimisation sur les essais en chambre de calibration

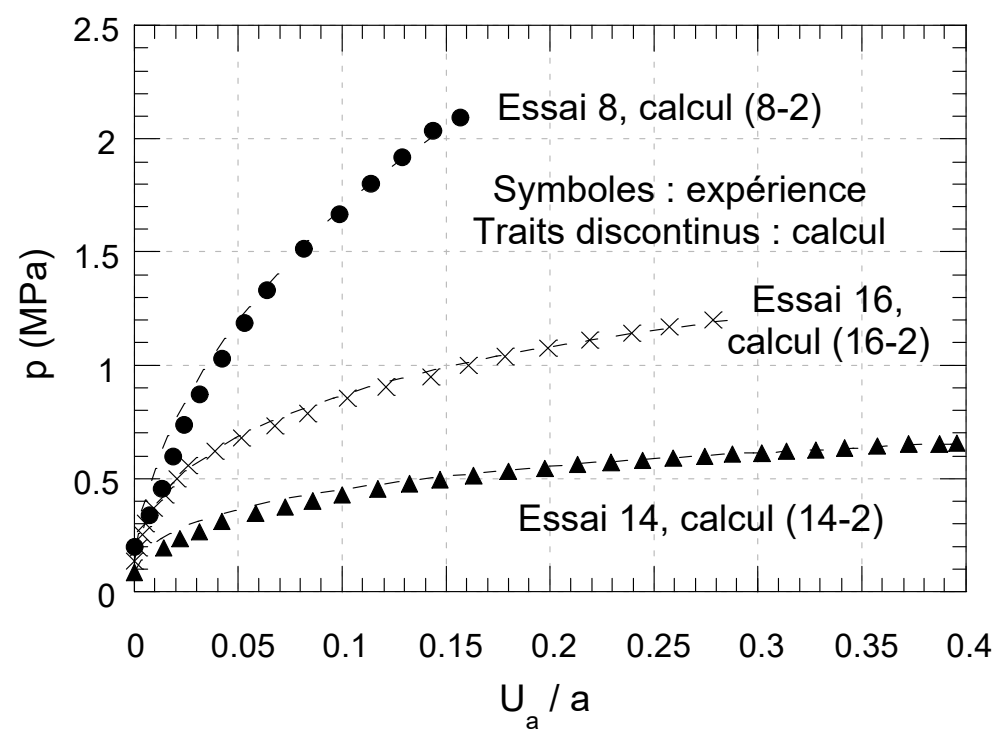

Figure 9. Résultats des calculs d'optimisation sur les essais en chambre de calibration

\subsection{Essais pressiométriques dans les sols injectés à Dunkerque}

On présente maintenant les résultats des calculs d'optimisation effectués, à partir de la formulation de (Yu et Houlsby, 1991), sur les essais pressiométriques réalisés sur le site de Dunkerque dans des sables fins injectés pour un traitement d'étanchéité. On a retenu trois essais représentatifs (figure 10) :

- deux essais conventionnels dans du sable avant injection, notés SP1-8 et SP2-8 à huit mètres de profondeur respectivement ; 
- deux essais conventionnels dans le sable après injection, notés SP1P-8 et SP2P-8 à huit mètres de profondeur, pour lesquels la pression des terres au repos est calculée conformément à la relation [16], avec un coefficient de pression des terres au repos $\mathrm{K}_{0}$ égal à l'unité. Les poids volumiques respectifs du sable non traité et du sable injecté sont respectivement de $19,6 \mathrm{kN} / \mathrm{m}^{3}$ et $21 \mathrm{kN} / \mathrm{m}^{3}$. La nappe est située à 5 mètres de profondeur. On considère que les pressions des terres au repos sont $: \mathrm{p}_{0 \mathrm{v}}=$ $127 \mathrm{kPa}$ et $\mathrm{p}_{0 \mathrm{i}}=138 \mathrm{kPa}$.

Les modules élastiques avant et après injection sont déduits de deux autres essais pressiométriques, avec cycle, réalisés dans les mêmes conditions à 5,5 et 5 mètres de profondeur. Les valeurs des modules de cisaillement $G_{v, \text { cycle }}$ et $G_{i, \text { cycle }}$ sont respectivement égales à $61 \mathrm{MPa}$ et $85 \mathrm{MPa}$. Pour les essais dans le sol non injecté, on corrige la valeur $\mathrm{G}_{\mathrm{v}, \mathrm{cycle}}$ de l'effet de la profondeur et de l'effet du niveau de contraintes lors du déchargement : on obtient un module élastique $\mathrm{E}_{\mathrm{v}}$ égal à $81 \mathrm{MPa}$. Pour les essais dans le sol injecté, on procède aux mêmes corrections puisque le coulis injecté est assez faiblement dosé en liant. Le module élastique $\mathrm{E}_{\mathrm{i}}$ est alors égal à $100 \mathrm{MPa}$. Le coefficient de Poisson est fixé à une valeur de 0,25 .

Pour le sable non injecté situé sous le niveau de la nappe, la cohésion est considérée comme nulle. L'angle de dilatance est corrélé à l'angle de frottement interne [Eq. 20]. Les calculs d'optimisation conduisent alors à des valeurs de l'angle de frottement interne de 39,8 degrés pour l'essai SP1-8 et 51,1 degrés pour l'essai SP2-8 (tableau 6, figure 10). Cette différence peut s'expliquer en partie par les perturbations du sol lors du forage.

Des calculs similaires réalisés avec le modèle en petites déformations [Eq. 14], au lieu du modèle en grandes déformations [Eq. 15] utilisé précédemment, conduisent à des valeurs de l'angle de frottement interne de 34,1 degrés pour l'essai SP1-8 et 41,0 degrés pour l'essai SP2-8.

Pour le sable injecté, on fixe la valeur de l'angle de frottement interne à 51,1 degrés pour l'essai SP1P-8 et à 39,8 degrés pour l'essai SP2P-8. L'optimisation simultanée de la cohésion c' et de l'angle de dilatance $\psi$ donne alors des valeurs $\mathrm{c}^{\prime}=282 \mathrm{kPa}$ et $\psi=38,8$ degrés pour l'essai SP1P-8 et c' $=20 \mathrm{kPa}$ et $\psi=29,7$ degrés pour l'essai SP2P-8 (tableau 6 ; figure 10 ).

On montre également l'influence de l'angle de frottement interne en considérant une valeur arbitraire de 45,3 degrés qui correspond à la moyenne des deux valeurs de 1'angle de frottement obtenues à partir des essais SP1-8 et SP2-8 (tableau 6). La valeur de l'angle de frottement interne semble plus affecter la valeur optimisée de l'angle de dilatance que celle de la cohésion.

$\mathrm{Au}$ stade actuel des recherches et des développements, l'analyse inverse des essais pressiométriques permet d'estimer la valeur de la cohésion des sols injectés et de proposer un intervalle de valeurs possibles qu'il convient de valider par des expériences en laboratoire. 


\begin{tabular}{||c|cccccc||}
\hline \hline Essai & $\begin{array}{c}\mathrm{p}_{0} \\
\mathrm{kPa}\end{array}$ & $\begin{array}{c}\mathrm{E} \\
\mathrm{MPa}\end{array}$ & $\nu$ & $\begin{array}{c}\varphi^{\prime} \\
\text { degrés }\end{array}$ & $\begin{array}{c}\psi \\
\text { degrés }\end{array}$ & $\begin{array}{c}\mathrm{c}^{\prime} \\
\mathrm{kPa}\end{array}$ \\
\hline SP1-8 & 127 & 81 & 0,25 & 39,8 & 9,8 & 0 \\
SP2-8 & 127 & 81 & 0,25 & 51,1 & 21,1 & 0 \\
\hline SP1P-8 & 138 & 100 & 0,25 & 51,1 & 38,8 & 282 \\
SP1P-8 & 138 & 100 & 0,25 & 45,3 & 45,1 & 265 \\
SP2P-8 & 138 & 100 & 0,25 & 39,8 & 29,7 & 20 \\
SP2P-8 & 138 & 100 & 0,25 & 45,3 & 24,6 & 7 \\
\hline \hline
\end{tabular}

Tableau 6. Calculs d'optimisation sur les essais de Dunkerque

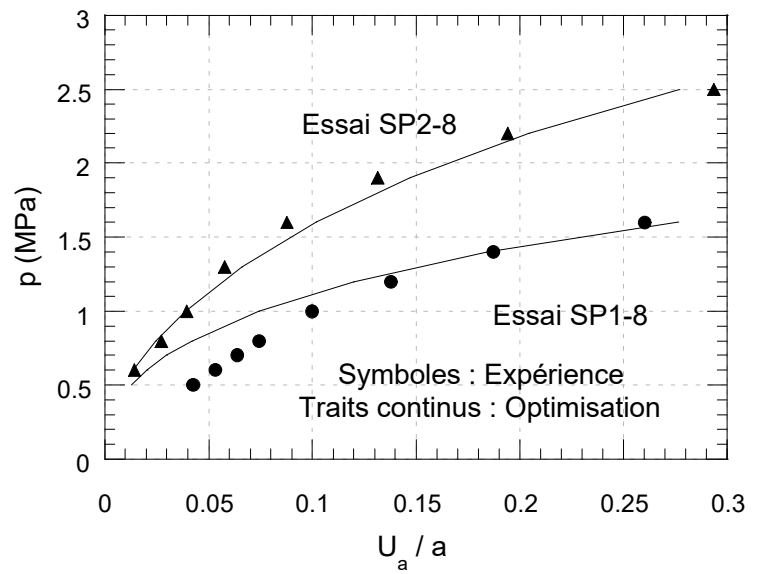

Avant injection

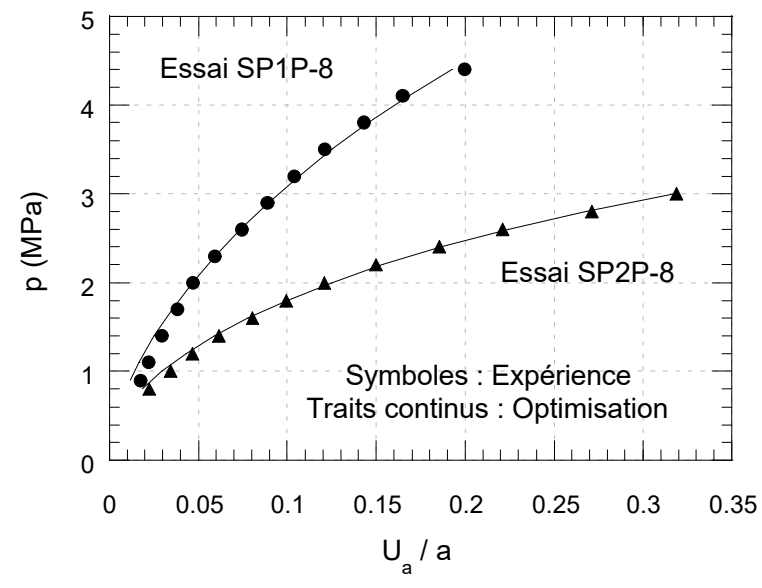

Après injection

Figure 10. Optimisation sur les essais pressiométriques de Dunkerque

\subsection{Comparaison avec les essais triaxiaux en laboratoire}

Des essais triaxiaux drainés ont été réalisés sur du sable de Dunkerque vierge et injecté prélevé in situ ou reconstitué en laboratoire (Tailliez, 1998 ; Dano, 2001). Le sable est mis en place à la densité in situ, ce qui correspond à un poids volumique de $15,7 \mathrm{kN} / \mathrm{m}^{3}$. Les résultats sont présentés sur la figure 11 .

On obtient, pour le sable vierge de tout traitement, un angle de frottement interne de 43 degrés et une cohésion nulle. On déduit également de l'évolution de la déformation volumique, de type contractant-dilatant, une valeur du coefficient de Poisson égale à 0,28 et un angle de dilatance compris entre 11,5 et 15,3 degrés en fonction de la contrainte de confinement variant de 50 à $400 \mathrm{kPa}$. Le module tangent à l'origine de la courbe contrainte-déformation vaut respectivement 37 et $59 \mathrm{MPa}$ pour des contraintes de confinement de 100 et $200 \mathrm{kPa}$ (tableau 7, figure 11).

S'il apparaît in situ que l'objectif d'étanchéité est bien atteint, l'injection engendre aussi une amélioration des propriétés mécaniques du terrain, en termes de rigidité et de résistance. Pour les échantillons de sol injecté prélevés in situ, le 
module tangent est compris entre 135 et $190 \mathrm{MPa}$. Dans le plan de Mohr, l'enveloppe intrinsèque est une droite à partir de laquelle on détermine la valeur de la cohésion (160 kPa) et la valeur de l'angle de frottement interne (41 degrés). On ne présente pas l'évolution de la déformation volumique car l'état de surface des échantillons ne permet pas de mesures précises (Tailliez, 1998).

On a aussi cherché à évaluer la validité du système d'injection en laboratoire en injectant, dans une colonne de PVC de un mètre de hauteur, le sable de Dunkerque par le coulis utilisé in situ. Les résultats des essais triaxiaux drainés sont présentés sur la figure 11. On observe que la résistance des échantillons préparés en laboratoire est systématiquement inférieure à celle des échantillons prélevés in situ. Comme l'angle de frottement interne est identique dans les deux cas (41 degrés), la diminution de la résistance se traduit par une diminution de la cohésion : celle-ci vaut $100 \mathrm{kPa}$ pour les échantillons injectés en laboratoire. Cette diminution peut s'expliquer par le mode d'injection différent dans les deux cas. En laboratoire, tous les paramètres, ceux liés au sol, au coulis ou à l'injection, sont parfaitement contrôlés : il s'ensuit une imprégnation homogène du sol par le coulis. Au contraire, l'injection in situ, réalisée au moyen de tubes à manchettes, nécessite un claquage préalable du coulis de gaine qui, s'il est mal contrôlé, se propage dans le sol, créant des zones où la concentration de coulis est plus élevée que celle souhaitée. On note également que le module tangent à l'origine de la courbe contrainte-déformation dépend relativement peu de la contrainte de confinement. Il vaut $140 \mathrm{MPa}$. On trouve un coefficient de Poisson de 0,21 et un angle de dilatance compris entre 16,8 et 21,4 degrés.

On constate donc que le comportement du sol injecté in situ est qualitativement identique à celui du sol injecté en laboratoire. Par ailleurs, les valeurs des paramètres obtenues pour les essais triaxiaux sont du même ordre de grandeur que celles calculées par la procédure d'optimisation.

\section{Commentaires}

L'interprétation des essais pressiométriques par analyse inverse dépend de leur représentativité, l'essai pressiométrique ne concernant qu'un petit volume d'un massif traité, et de la qualité de leur exécution. L'étude de sensibilité de la courbe pressiométrique aux paramètres du modèle permet de comprendre que le moindre défaut, en particulier le remaniement provoqué par le forage, peut conduire à des résultats aberrants. Pour ces raisons, l'utilisation d'un pressiomètre auto-foreur est préférable. Mais il ne faut pas imputer toutes les erreurs à l'exécution des essais pressiométriques. La procédure, les hypothèses de calcul (l'élancement de la sonde et l'influence de la contrainte intermédiaire ne sont pas pris en compte par exemple) et le modèle choisi ne sont pas exempts de critiques. 

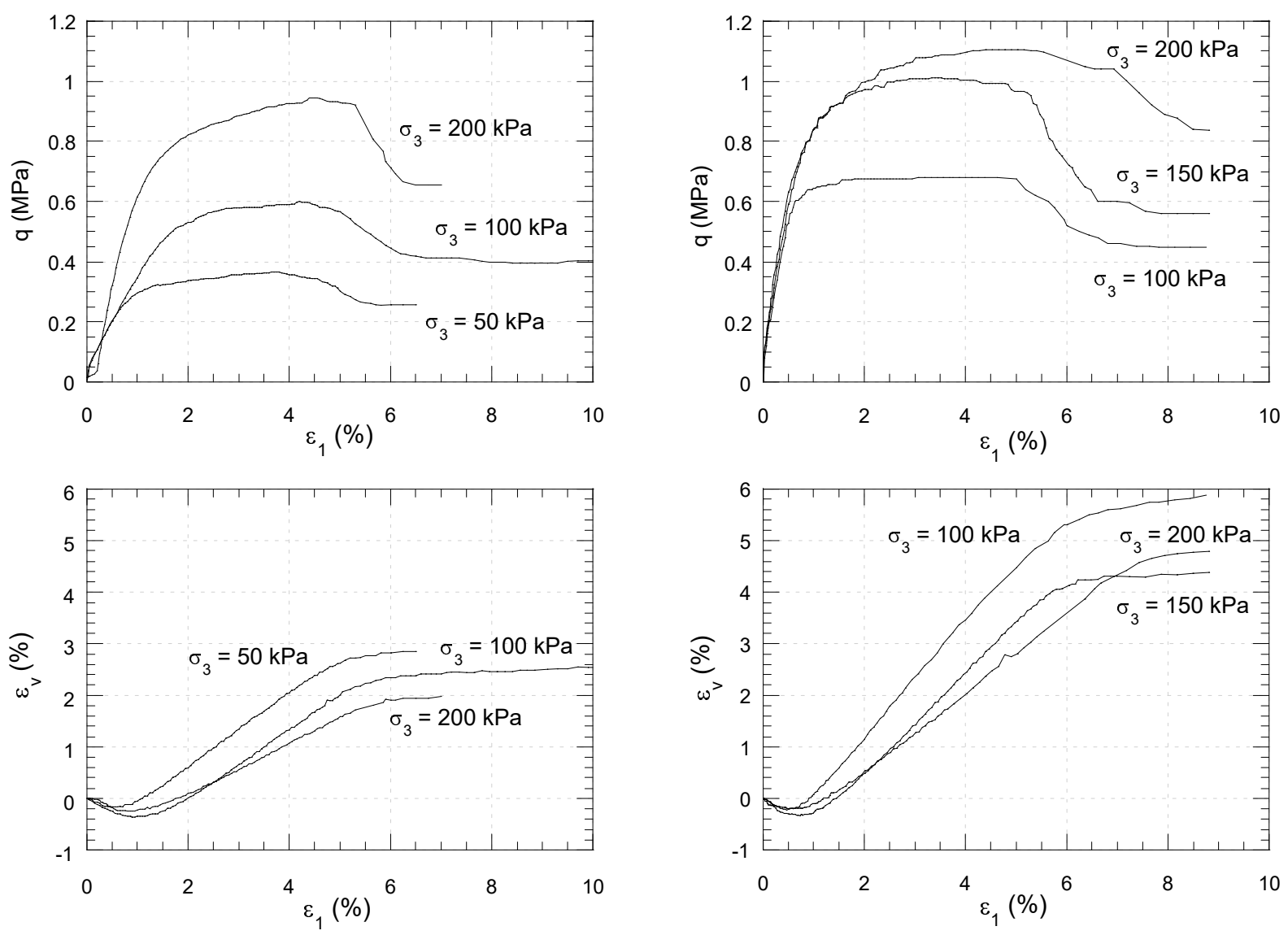

Sable de Dunkerque vierge.

Sable de Dunkerque injecté en laboratoire.

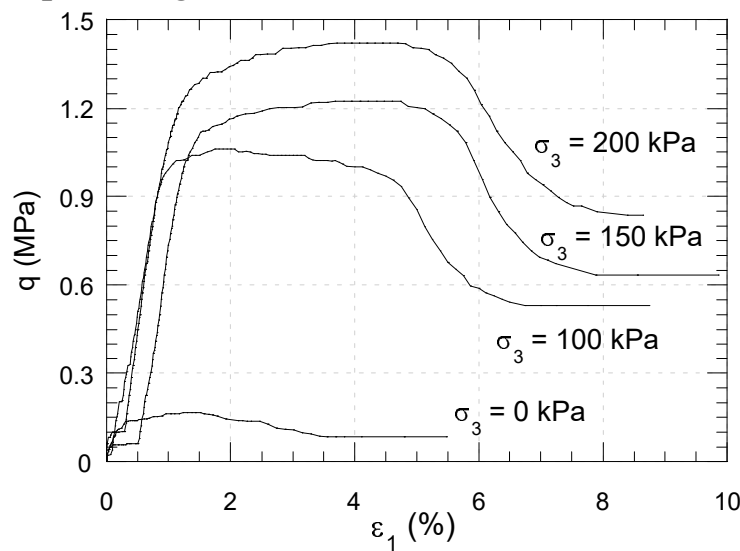

Sable de Dunkerque injecté in situ.

Figure 11. Essais triaxiaux drainés sur le sable de Dunkerque vierge et injecté (Tailliez, 1998)

\begin{tabular}{||c|ccccc||}
\hline $\begin{array}{c}\text { Matériau } \\
100 \leq \sigma_{3}^{\prime} \leq 400 \mathrm{kPa}\end{array}$ & $\begin{array}{c}\mathrm{E} \\
\mathrm{MPa}\end{array}$ & $v$ & $\begin{array}{c}\varphi^{\prime} \\
\text { degrés }\end{array}$ & $\begin{array}{c}\psi \\
\text { degrés }\end{array}$ & $\begin{array}{c}\mathrm{c}^{\prime} \\
\mathrm{kPa}\end{array}$ \\
\hline Sable vierge & 37 à 59 & 0,28 & 42 & 11,5 à 15,3 & 0 \\
Sable injecté in situ & 135 à 190 & - & 41 & - & 160 \\
Sable injecté en laboratoire & 140 & 0,21 & 41 & 16,8 à 21,4 & 100 \\
\hline
\end{tabular}

Tableau 7. Paramètres du sable de Dunkerque déterminés en laboratoire 


\subsection{Représentation de l'enveloppe intrinsèque dans le domaine des contraintes de traction}

On a supposé que l'enveloppe intrinsèque était linéaire jusque dans le domaine des contraintes de traction. Cette hypothèse n'est pas justifiée expérimentalement (tableau 8). (Tailliez, 1998) a ainsi entrepris des essais de traction brésiliens (résistance $\mathrm{R}_{\mathrm{TB}, \mathrm{exp}}$ ) et des essais de traction directe (résistance $\mathrm{R}_{\mathrm{TD} \text {,exp }}$ ) sur des éprouvettes de sable de Fontainebleau injecté par un coulis à base de ciment fine mouture et par un coulis minéral, tous deux déclinés sous trois formulations à teneur en liant croissante. La résistance à la traction directe vaut respectivement $1,25 \%$ et $2 \%$ de la résistance en compression simple $\mathrm{R}_{\mathrm{c}}$ pour le coulis de ciment et le coulis minéral tandis que la résistance à la traction théorique donnée par l'équation [22] est comprise entre 20 et $25 \%$ de la résistance en compression simple pour des valeurs usuelles des paramètres du modèle de Mohr-Coulomb linéaire :

$$
\mathrm{R}_{\mathrm{TD}, \text { theo }}=\frac{2 \mathrm{c}^{\prime} \cdot \cos \varphi^{\prime}}{1+\sin \varphi^{\prime}}
$$

Ceci signifie que le critère de rupture doit être considéré comme incurvé dans le domaine des contraintes de traction et pourrait être approché par une équation de type parabolique (Ladanyi, 1976).

\begin{tabular}{|c|c|c|c|c|c|c|c|}
\hline \multirow{2}{*}{\multicolumn{2}{|c|}{ Formulation }} & \multicolumn{3}{|c|}{ Coulis de ciment CC } & \multicolumn{3}{|c|}{ Coulis minéral CM } \\
\hline & & 1 & 2 & 3 & 1 & 2 & 3 \\
\hline $\mathrm{R}_{\mathrm{c}}$ & $\mathrm{MPa}$ & 0,9 & 1,1 & 1,9 & 0,6 & 0,9 & 1,9 \\
\hline $\mathrm{R}_{\mathrm{TB}, \exp } / \mathrm{R}_{\mathrm{c}}$ & $\%$ & 17 & 23 & 16 & 8,3 & 7,8 & 6,8 \\
\hline $\mathrm{R}_{\mathrm{TD}, \exp } / \mathrm{R}_{\mathrm{c}}$ & $\%$ & \multicolumn{3}{|c|}{1 à 1,25} & \multicolumn{3}{|c|}{1,7 à 2} \\
\hline $\mathrm{R}_{\mathrm{TD} \text {, theo }} / \mathrm{R}_{\mathrm{c}}$ & $\%$ & \multicolumn{3}{|c|}{20 à 25} & \multicolumn{3}{|c|}{20 à 25} \\
\hline
\end{tabular}

Tableau 8. Résistance à la traction du sable de Fontainebleau injecté

\subsection{La question de la rupture par fissuration}

Cette résistance à la traction est la traduction à l'échelle de l'échantillon de la cimentation des grains entre eux provoquée par la prise du liant. Par ailleurs, les essais de compression triaxiale en laboratoire ont montré, suivant la valeur de la contrainte latérale et de la teneur en ciment du coulis, une rupture par localisation avec apparition de fissures verticales. Ces deux constatations amènent à aborder la question de la fissuration, plus généralement de l'endommagement du matériau, et 
de la prépondérance de ce mécanisme dans la réponse du matériau à une sollicitation de type pressiométrique.

Les modèles élasto-plastiques présupposent une rupture par compression, fonction de l'angle de frottement interne et de la cohésion. Or, comme indiqué dans la littérature pour des roches au comportement élastique linéaire (Haberfield et Johnston, 1990 ; Haberfield, 1997), la sollicitation pressiométrique peut engendrer des déformations d'extension dans le sens orthoradial, si bien que des fissures peuvent apparaître au droit $\mathrm{du}$ forage et se propager dans le sol, modifiant complètement le mode de rupture et la distribution des contraintes dans le sol. Une telle rupture peut se produire lorsque la pression dans la cavité atteint la valeur $p_{t}$ (Cambefort, 1997) telle que :

$$
\mathrm{p}_{\mathrm{t}}=2 \mathrm{p}_{0}+\mathrm{R}_{\mathrm{TD}}
$$

où $\mathrm{R}_{\mathrm{TD}}$ est la résistance à la traction du matériau. Aussi, l'analyse élasto-plastique peut être remise en cause si $\mathrm{p}_{\mathrm{t}}<\mathrm{p}_{\mathrm{pl}}$, auquel cas la fissuration se produit avant la rupture par compression. Si l'on adopte le critère parabolique proposé par (Ladanyi, 1976) et que l'on introduit les données expérimentales du tableau 8, les valeurs de la pression des terres au repos sont compatibles avec le développement de la fissuration. Aussi, en toute rigueur, le modèle de comportement doit donc permettre de prédire l'initiation des fissures, leur propagation dans le sens radial et leur refermeture par suite du développement des zones plastiques entre ces fissures (Haberfield et Johnston, 1990).

La simulation de l'essai pressiométrique avec un modèle basé sur la mécanique de la rupture, introduit dans un code de calcul par éléments finis, montre, pour une pression dans la sonde donnée, une déformation a la paroi accentuée en considérant la fissuration. Toutefois, ce mécanisme n'est prépondérant que dans le cas d'essais pressiométriques à faible profondeur et dans la partie initiale de la courbe pressiométrique, jusqu'à une déformation à la paroi de l'ordre de 2 à $3 \%$. En dehors de ces conditions, le mécanisme élasto-plastique est prédominant (Haberfield et Johnston, 1990).

\subsection{La question du radoucissement du matériau}

Un modèle plastique parfait non associé engendre une déformation volumique non limitée. Or, les essais de laboratoire ont montré une phase de radoucissement, postérieure au pic de contraintes de cisaillement, conduisant à un palier du déviateur des contraintes et de la déformation volumique. Ce radoucissement, comme précédemment pour la fissuration, s'accompagne d'une redistribution des contraintes affectant le mode de sollicitation du sol et la courbe pressiométrique. (Carter et Yeung, 1985) montre que, dans le cas d'un sol purement frottant (à dilatance nulle), la pression limite dépend du taux de radoucissement en fonction de 
la déformation plastique. Une valeur maximale est obtenue dans le cas du modèle élasto-plastique parfait sans radoucissement. La prise en compte du radoucissement conduit la courbe pressiométrique à se rapprocher de celle obtenue en considérant non plus les caractéristiques au pic de contraintes mais les caractéristiques dans l'état de plasticité parfaite (Dano, 2001).

\subsection{Paramètres élastiques}

Les valeurs du module tangent déterminées soit par des essais triaxiaux conventionnels, soit par les cycles de déchargement-rechargement en cours d'essais pressiométriques à des niveaux de déformations compris entre $0,1 \%$ et $1 \%$, restent très en deçà des valeurs mesurées dans le domaine des petites déformations, en raison de la non-linéarité du comportement des sols. Deux dispositifs ont été utilisés pour déterminer les propriétés élastiques des sols injectés dans le domaine des petites déformations. Le premier consiste à émettre des ondes ultrasonores unidirectionnelles au travers de l'échantillon et à calculer leur vitesse de propagation V (tableau 9). Le second, appelé GrindoSonic (Allison, 1988), consiste à exciter l'échantillon par un choc élastique, suivant deux modes de sollicitation distincts (flexion et torsion) et à recueillir la réponse vibratoire qui en résulte. On détermine la fréquence de résonance puis on calcule les caractéristiques élastiques (tableau 9). On fait l'hypothèse d'un comportement élastique linéaire a priori justifié dans le domaine des déformations concernées $\left(\varepsilon<10^{-6}\right)$ pour remonter aux valeurs du module d'Young, du module de cisaillement et du coefficient de Poisson. On donne, dans le tableau 9, des valeurs des caractéristiques élastiques mesurées, à confinement nul, sur des échantillons de sables injectés en laboratoire. Elles sont d'un ordre de grandeur supérieure aux valeurs introduites dans les calculs d'optimisation.

\subsection{Conditions de drainage}

L'essai pressiométrique est-il drainé ou non drainé ? Cette question revient régulièrement dans les discussions sur l'interprétation de cet essai (Haberfield et Johnston, 1990 ; Cambou et Bahar, 1993 ; Bolton et Whittle, 1999). Dans le cas des sols injectés, la question est d'importance puisque la perméabilité du terrain est radicalement modifiée par l'injection, en particulier pour un traitement d'étanchéité. Avant injection, l'essai pressiométrique, sauf conditions locales particulières, est nécessairement drainé car les sols susceptibles d'être injectés par des coulis de ciment fine mouture ont une perméabilité comprise entre $10^{-3}$ et $10^{-6} \mathrm{~m} / \mathrm{s}$. Après injection, la perméabilité du sol est réduite et peut être ramenée à des valeurs comprises entre $10^{-6}$ et $10^{-9} \mathrm{~m} / \mathrm{s}$. Si l'on considère l'essai comme non drainé, alors il faut pouvoir modéliser l'évolution des pressions interstitielles qui, si elle n'est pas prise en compte, peut conduire à une mauvaise estimation des paramètres de comportement par analyse inverse. 


\begin{tabular}{|c|c|c|ccc|c||}
\hline Matériau & $\mathrm{C} / \mathrm{E}$ & $\begin{array}{c}\gamma \\
\mathrm{kN} / \mathrm{m}^{3}\end{array}$ & $\begin{array}{c}\mathrm{G} \\
\mathrm{GPa}\end{array}$ & $\begin{array}{c}\mathrm{E} \\
\mathrm{GPa}\end{array}$ & $v$ & $\begin{array}{c}\mathrm{V} \\
\mathrm{m} / \mathrm{s}\end{array}$ \\
\hline Sable Fontainebleau & 0,172 & 20,4 & 3,9 & 9,1 & 0,18 & 2640 \\
Sable Fontainebleau & 0,235 & 20,6 & 5,2 & 12,4 & 0,18 & 2895 \\
Sable Fontainebleau & 0,299 & 20,7 & 6,1 & 15,7 & 0,22 & 3087 \\
Alluvions 1 & 0,172 & 20,6 & 4,4 & 11,1 & 0,20 & 2778 \\
Alluvions 2 & 0,172 & 21,6 & 5,2 & 11,9 & 0,14 & 2790 \\
\hline
\end{tabular}

Tableau 9. Propriétés élastiques en petites déformations

\section{Conclusions}

Jusqu'à présent, l'amélioration mécanique apportée par un traitement d'injection n'a que rarement été prise en compte de manière rationnelle dans les calculs de soutènements d'ouvrages souterrains, les coefficients de sécurité étant largement surestimés. Après avoir rappelé les principaux traits relatifs au comportement des sables injectés, on indique dans ce document des valeurs des facteurs d'amélioration des paramètres d'un modèle de comportement élastique linéaire-plastique parfait avec un critère de Mohr-Coulomb et avec une loi d'écoulement non associée.

On propose aussi une procédure d'interprétation des essais pressiométriques in situ dans le but d'identifier les valeurs des paramètres de ce modèle. Cette procédure fait appel à la technique de l'analyse inverse de la courbe pressiométrique expérimentale en considérant la formulation analytique de $\mathrm{Yu}$ et Houlsby, en grandes déformations, et un algorithme d'optimisation de Newton-Gauss, l'ensemble étant facilement implanté dans un tableur. Cette solution technique donne des résultats satisfaisants compte tenu des hypothèses de calcul.

\section{Bibliographie}

Allison R.J., «A non-destructive method of determining rock strength», Earth Surface Processes and Landforms, vol. 13, 1988, p. 1-8.

Baguelin F., Jézéquel J.F., Shields D.H., The pressuremeter and foundation engineering, Trans. Tech Publications, 1978.

Biarez J., David H., Gouvenot D., Hicher P.Y., Tailliez S.,Varjabedian M., « Comportement mécanique des sols granulaires traités par injection », Revue française de génie civil, vol. $2, \mathrm{n}^{\circ} 2,1998$, p. 255-295.

Bellotti R., Ghionna V., Jamiolkowski M., Robertson P.K., Peterson R.W., «Interpretation of moduli from self-boring pressuremeter tests in sand », Geotechnique, vol. 39, n 2, 1989, p. 269-292. 
Bolton M.D., « The strength and dilatancy of sands », Geotechnique, vol. 36, $\mathrm{n}^{\circ}$ 1, 1986, p. $65-78$.

Bolton M.D., Whittle R.W., «A non linear elastic/perfectly plastic analysis for plane strain undrained expansion tests », Geotechnique, vol. 49, n 1, 1999, p. 133-141.

Cambefort H., L’injection des sols, Editions Eyrolles, 1967.

Cambefort H., "Les claquages dans l'injection des sols », Annales du Bâtiment et des Travaux Publics, Sols et Fondations, juin 1997, p. 61-70.

Cambou B., Bahar R., «Utilisation de l'essai pressiométrique pour l'identification de paramètres intrinsèques du comportement d'un sol », Revue Française de Géotechnique, $\mathrm{n}^{\circ} 63,1993$, p. 39-50.

Carter J.P., Yeung S.K., «Analysis of cylindrical cavity expansion in a strain weakening material », Computers and Geotechnics, $\mathrm{n}^{\circ} 1,1985$, p. 161-180.

Carter J.P., Booker J.R., Yeung S.K., « Cavity expansion in cohesive frictional soils », Geotechnique, vol. 36, n 3, 1986, p. 349-358.

Clough G.W., Sitar B., « Cemented sand under static loading », Journal of the Geotechnical Engineering, vol. 107, n GT6, 1981, p. 799-817.

Combarieu O., "L'essai pressiométrique et la résistance au cisaillement des sols », Bulletin de Liaison des Laboratoires des Ponts et Chaussées, n 196, Article 3886, 1995.

Dano C., Comportement mécanique des sols injectés, Thèse de Doctorat, Ecole Centrale de Nantes \& Université de Nantes, 2001.

Dupas J.M., Pecker A., "Static and dynamic properties of sand-cement », Journal of the Geotechnical Engineering Division, Proceedings of the American Society of Engineers, vol. 105, n GT3, 1979, p. 419-436.

Haberfield C.M., Johnston I.W., «A numerical model for pressuremeter testing in soft rock », Geotechnique, vol. 40, n 4, 1990, p. 569-580.

Haberfield C.M., « Pressuremeter testing in weak rock and cemented sand », Proceedings of the Institution of Civil Engineers - Geotechnical Engineering, vol. 125, juillet 1997, p. 168-178.

Hughes J.M.O., Wroth C.P., Windle D., « Pressuremeter tests in sands », Geotechnique, vol. $27, \mathrm{n}^{\circ} 4,1977$, p. $455-477$.

Kasdi A., Détermination des paramètres des modèles élasto-plastiques à partir de l'essai pressiométrique, Thèse de Doctorat, Université des Sciences et Technologies de Lille, 1994.

Ladanyi B., "Quasi-static expansion of a cylindrical cavity in rock », 3rd Symposium on engineering applications of solid mechanics, Toronto, vol. 3, 1976, p. 219-240. 
Lee K.L., "Comparison of plane strain and triaxial tests on sand », Journal of the Soil Mechanics and Foundations Division, vol. 96, n SM3, 1970, p. 901-923.

Mestat P., «Loi de Mohr-Coulomb », Revue française de génie civil, vol. 1, n 1, 1997, p. 189-192.

Mokrani L., Simulation physique du comportement des pieux à grande profondeur en chambre de calibration, Thèse de Doctorat, Institut National Polytechnique de Grenoble, 1991.

Monnet J., Khlif J., «Etude théorique de l'équilibre élasto-plastique d'un sol pulvérulent autour du pressiomètre », Revue Française de Géotechnique, n 67, 1994, p. 3-12.

Monnet J., Chemaa T., «Etude théorique et expérimentale de l'équilibre élasto-plastique d'un sol cohérent autour du pressiomètre », Revue Française de Géotechnique, ${ }^{\circ} 73$, 1995, p. 15-26.

Schnaid F., Mántaras F.M., «Assessment of soil properties in cohesive-frictional materials with pressuremeter tests », Geotechnical Site Characterization, Proceedings of the 1st International Conference on site characterization, vol. 2, Atlanta, 1998.

Shahrour I., Kasdi A., Abriak N., «Utilisation de l'essai pressiométrique pour la détermination des propriétés mécaniques des sables obéissant au critère de MohrCoulomb avec une règle d'écoulement non associée », Revue Française de Géotechnique, $\mathrm{n}^{\circ} 73,1995$, p. $27-33$.

Shao J.F., Dahou A., Henry J.P., «Application de la théorie des problèmes inverses à l'estimation des paramètres des modèles rhéologiques ", Revue Française de Géotechnique, $\mathrm{n}^{\circ}$ 57, 1991, p. 75-80.

Tailliez S., Etude expérimentale du comportement mécanique des sols granulaires injectés, Thèse de Doctorat, Ecole Centrale de Paris, 1998.

Vaid Y.P., Sasitharan S., « The strength and dilantancy of sands », Canadian Geotechnical Journal, vol. 29, 1992, p. 522-526.

Yu H.S., Houlsby G.T., «Finite cavity expansion in dilatant soils: loading analysis », Geotechnique, vol. 41, n² 2, 1991, p. 173-183.

Zentar R., Analyse inverse des essais pressiométriques - Application à l'argile de SaintHerblain, Thèse de Doctorat, Ecole Centrale de Nantes. 1999.

GrindoSonic, Manuel de l'utilisateur.

Fascicule $\mathrm{n}^{\circ} 62$, Règles techniques de conception et de calcul des fondations des ouvrages de génie civil.

Norme Française NF P 94-110-1 : Sols - Reconnaissance et essais - Essai pressiométrique Ménard - Essai sans cycle, 1991.

Norme Française NF P 94-110-2 : Sols - Reconnaissance et essais - Essai pressiométrique Ménard - Essai avec cycle, 1999. 